\title{
STEEPEST-DESCENTS INTEGRAL REPRESENTATIONS FOR DOMINANT SOLUTIONS OF LINEAR SECOND-ORDER DIFFERENTIAL EQUATIONS
}

\author{
William G. C. Boyd
}

\begin{abstract}
It is shown that for certain linear second-order differential equations, there are solutions (usually referred to as recessive solutions) which have integral representations of Stieltjes transform type. It also is shown that there are special dominant solutions (the solution Bi of Airy's equation is an archetype) which have integral representations of one-sided Hilbert transform type. The differential equations we consider are those whose general solution may be expressed as an integral to which the method of steepest descents is applicable. Berry and Howls' formulation of the method of steepest descents is used to discuss the asymptotic properties of both kinds of solution. Our work suggests that the solutions of linear second-order differential equations in the neighbourhood of a Stokes line can be distinguished by the behavior of their remainders after the contributions from the dominant terms (up to optimal truncation) in their asymptotic approximations have been removed.
\end{abstract}

\section{Introduction}

The method of steepest descents is applicable to integrals of the form

$$
\int_{C} \mathrm{e}^{-z p(w)} q(w) \mathrm{d} w
$$

for $|z| \rightarrow \infty$. In the standard application of the method, one first finds the saddle points of $p(w)$ (the zeros of $p^{\prime}(w)$ ) and then deforms the contour $C$ into a path of steepest descents through one of the saddle points, or into a combination of such paths. We assume that $p(w)$ and $q(w)$ are holomorphic functions and that $p^{\prime}(w)$ has only simple zeros, located at $w^{(1)}, w^{(2)}, \ldots$; we assume that there are at least two such zeros at which $p(w)$ takes distinct values. Let us consider $Y^{(n)}(z)$, the integral over the path of steepest descents $C^{(n)}(\theta)$ through the saddle point $w^{(n)}$ :

$$
Y^{(n)}(z)=\int_{C^{(n)}(\theta)} \mathrm{e}^{-z p(w)} q(w) \mathrm{d} w .
$$

Here, and subsequently, $\theta$ denotes $\operatorname{ph}(z)$ : the paths of steepest descents $C^{(n)}(\theta)$ through $w^{(n)}$, of course, change as $\theta$ varies. It is well-known that the value of an integral in the form (2) is increasingly dominated by the contribution from the neighbourhood of the saddle point as $|z| \rightarrow \infty$ : the asymptotic behavior is

$$
Y^{(n)}(z) \sim \frac{\mathrm{e}^{-z p^{(n)}}}{z^{\frac{1}{2}}} \sum_{r=0}^{\infty} \frac{a_{r}}{z^{r}}
$$

Received May 31, 1995, revised January 18, 1996.

1991 Mathematics Subject Classification: 34E05, 41A60, 41A80.

Key words and phrases: steepest descents, asymptotic expansions, Stokes' phenomenon. 
where $p^{(n)}=p\left(w^{(n)}\right)$, and that branch of the square root $z^{\frac{1}{2}}$ is chosen for which $z^{\frac{1}{2}}>0$ when $z>0$. The coefficients $a_{r}$ may be determined in terms of the properties of $p(w)$ and $q(w)$ at $w=w^{(n)}$. Accounts of the method may be found in the standard textbooks: see, for example, de Bruijn [13], Jeffreys [17], Copson [11], Olver [19], Bleistein and Handelsman [5], Bender and Orszag [2], or Wong [24].

Berry and Howls [4] have recently reformulated the method of steepest descents; to avoid unnecessary repetition, we shall refer to the account of their work given in Boyd [7]. We use the notation and nomenclature of that paper without further explanation; we also shall assume that the functions $p(w)$ and $q(w)$ which appear in the integrand of (1) satisfy conditions given there (Boyd [7], p. 503, Conditions 3.2).

Berry and Howls considered the slowly-varying factor $S^{(n)}(z)$ associated with $Y^{(n)}(z)$, defined by

$$
Y^{(n)}(z)=\frac{\mathrm{e}^{-z p^{(n)}}}{z^{\frac{1}{2}}} S^{(n)}(z)
$$

(compare (3) above), and showed that under a wide variety of circumstances (Boyd [7], p. 502, Theorem 3.1),

$$
S^{(n)}(z)=\frac{z}{2 \pi i} \int_{0}^{\infty} u^{-\frac{1}{2}} \mathrm{e}^{-u} \sum_{m} \int_{C^{(m)}} \frac{q(w)\left[p(w)-p^{(n)}\right]^{\frac{1}{2}}}{z\left[p(w)-p^{(n)}\right]-u} \mathrm{~d} w \mathrm{~d} u
$$

where the summation in question is over the adjacent saddle points $w^{(m)}$ : those saddle points which can be linked to $w^{(n)}$ by a path of steepest descents for some value of $\theta$. We refer to the contours $C^{(m)}$ as the adjacent contours (see Figure 1). Note that the orientation of the adjacent contours is specified by the requirement that they be traversed anti-clockwise with respect to $w^{(n)}$. The square root $\left[p(w)-p^{(n)}\right]^{\frac{1}{2}}$ in (5) is in fact single-valued and is defined to be positive on $C_{+}^{(n)}(0)$ : that half of the steepestdescents path $C^{(n)}$ which runs from the saddle point $w^{(n)}$ to $\infty$ when $\theta=0$. Berry and Howls showed that (5) could be expressed as (Boyd [7], p. 503, Theorem 3.3)

$$
S^{(n)}(z)=\frac{1}{2 \pi i} \sum_{m} \int_{0}^{\infty} \frac{v^{-1} \mathrm{e}^{-v} S^{(m)}\left(v / p^{(n m)}\right)}{1-v / z p^{(n m)}} \mathrm{d} v
$$

where $p^{(n m)}=p^{(m)}-p^{(n)}$ and $S^{(m)}(\eta)$ is defined by analogy with $S^{(n)}(z)$ : the corresponding integral over the adjacent contour $C^{(m)}$.

In this paper, we shall study the special features of integral representations of the form (1), which are also solutions of linear second-order differential equations. For this purpose, we find it preferable to consider the solution $Y^{(n)}(z)$ itself, rather than its slowly-varying factor $S^{(n)}(z)$. In $\S 2$, we derive the integral representation of Stieltjes transform type for $Y^{(n)}(z)$, analogous to (6), find analytic continuation formulae for $Y^{(n)}(z)$, and state other properties of $Y^{(n)}(z)$. Then in $\S 3$, we derive special results which hold good when integral representations of the form (1) satisfy linear second-order differential equations. In particular, in $\S 3.3$ we define a special dominant solution, which we denote by $y^{(n)}(z)$ and refer to as "the solution of $\mathrm{Bi}$ type associated with a Stokes line": we shall find that its asymptotic expansion has characteristic, and interesting, behavior at optimal truncation. In $\S 4$ and $\S 5$, we illustrate the general results of $\S 2$ and $\S 3$ by considering the solutions of the Airy equation and the modified Bessel equation respectively. Finally, in $\S 6$ we discuss the wider significance of the results: we suggest that the solutions of linear second-order differential equations in the neighbourhood of a Stokes line can be distinguished by the behavior of their remainders after the contributions from the dominant terms (up 

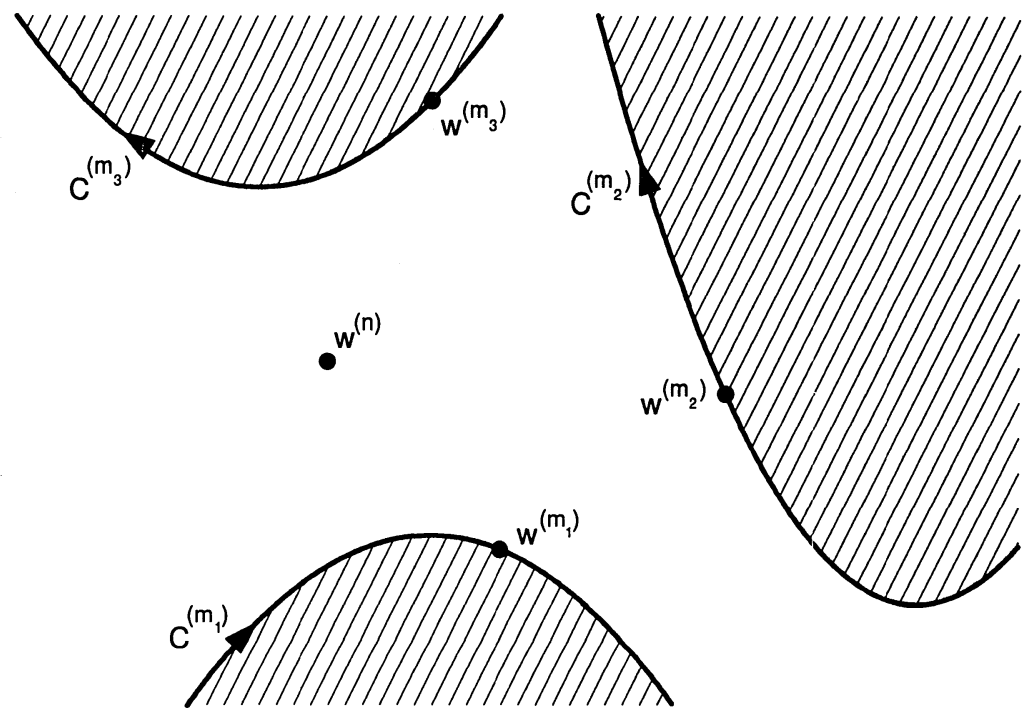

Figure 1. A typical domain associated with the saddle point $w^{(n)}$. (The domain is shown unshaded.) The contours on the boundary of the domain-the figure depicts $C^{\left(m_{1}\right)}, C^{\left(m_{2}\right)}, C^{\left(m_{3}\right)}$, passing through the adjacent saddle points $w^{\left(m_{1}\right)}, w^{\left(m_{2}\right)}, w^{\left(m_{3}\right)}$, respectively-are the adjacent contours.

to optimal truncation) in their asymptotic approximations have been removed. In the Appendix, we reformulate Olver's [21] results on the terminant function $F_{p}(x)$, and then introduce, and derive results for, another terminant function, $\bar{F}_{p}(x)$, which is necessary for our analysis.

\section{The integral representation for $Y^{(n)}(z)$}

2.1. Derivation of the representation. In the analysis used by Berry and Howls [4], and in its description by Boyd [7], the function $S^{(n)}(z)$, defined by (2) and (4) above, is taken to be an integral over a path of steepest descents for all values of $z$. As $\theta$ varies, the path of steepest descents through the saddle point $w^{(n)}$ suddenly changes discontinuously when $\theta=-\theta^{(n m)}$ modulo $2 \pi$, where $\theta^{(n m)}=\operatorname{ph}\left(p^{(n m)}\right)$. Clearly, therefore, $S^{(n)}(z)$ represents different analytic functions in different sectors of the $z$-plane. This had advantages in the approach used by Berry and Howls. For example, formula (6) is valid for all values of $\theta$ (except for the critical values $-\theta^{(n m)}$, at which the integrals are not uniquely defined). However, for our purposes in this paper, we prefer to use functions which can be continued analytically as $\theta$ varies. This, therefore, suggests that we should consider $Y^{(n)}$ rather than $S^{(n)}$. Moreover, in the context of linear differential equations, there are obvious advantages in using the solutions themselves (for instance, because connection formulae are then relatively simple), rather than specially chosen features of the solutions. 
We therefore shall focus our attention on $Y^{(n)}(z)$, and regard it as specified by the integral representation (2) for some value of $\theta$, say $\theta=\theta_{0}$, and for other values of $\theta$ by analytic continuation.

Likewise (with $\eta$ replacing $z$, and $C^{(m)}$ replacing $C^{(n)}$ ), we shall regard $Y^{(m)}(\eta)$ as specified by the integral representation $(2)$ for $\operatorname{ph}(\eta)=-\theta^{(n m)}$, and for other values of $\operatorname{ph}(\eta)$ by analytic continuation. To specify $-\theta^{(n m)}$, we shall distinguish between the adjacent points on each side of $C^{(n)}\left(\theta_{0}\right)$. With account taken of the orientation of $C^{(n)}\left(\theta_{0}\right)$, we can refer unambiguously to the adjacent saddle points on the right and the left. Then, if $w^{(m)}$ is a saddle point on the right, we specify that $-\theta^{(n m)}$ lies in the range

$$
\theta_{0}<-\theta^{(n m)}<\theta_{0}+2 \pi,
$$

while if it is on the left, we specify

$$
\theta_{0}-2 \pi<-\theta^{(n m)}<\theta_{0} .
$$

With $Y^{(n)}$ and $Y^{(m)}$ thus specified, we may readily express $(6)$ in terms of $Y^{(n)}$ and $Y^{(m)}$ :

$$
Y^{(n)}(z)=\frac{\mathrm{e}^{-z p^{(n)}}}{z^{\frac{1}{2}}} \frac{1}{2 \pi i} \sum_{m} \frac{1}{\left[p^{(n m)}\right]^{\frac{1}{2}}} \int_{0}^{\infty} \frac{v^{-\frac{1}{2}} \exp \left(v p^{(n)} / p^{(n m)}\right) Y^{(m)}\left(v / p^{(n m)}\right)}{1-v / z p^{(n m)}} \mathrm{d} v
$$

valid for values of $\theta$ near $\theta_{0}$. The square root $\left[p^{(n m)}\right]^{\frac{1}{2}}$ in $(9)$ is by definition $\left[p\left(w^{(m)}\right)-\right.$ $\left.p^{(n)}\right]^{\frac{1}{2}}$, and its branch, therefore, is specified by our discussion just above (6): that is, the single-valued function $\left[p(w)-p^{(n)}\right]^{\frac{1}{2}}$ is positive on $C_{+}^{(n)}(0)$, and is defined elsewhere by analytic continuation. It should be noted that, of the two possible branches of the square root $\left[p(w)-p^{(n)}\right]^{\frac{1}{2}}$, the one which is specified by our definition depends on the specific orientation of the original contour $C^{(n)}\left(\theta_{0}\right)$ and the specific choice of $\theta_{0}$.

2.2. Analytic continuation formulae. Consider the effect of allowing $\theta$ to increase from its original value $\theta_{0}$ in (9). As $\theta$ passes through the values $-\theta^{(n m)}$, the poles $v=$ $z p^{(n m)}$ are captured: the residues after capture are either $+Y^{(m)}(z)$ or $-Y^{(m)}\left(z \mathrm{e}^{-2 \pi i}\right)$. This, perhaps, is most easily seen by considering the effect of allowing $\theta$ to increase in the representation (2) for $Y^{(n)}(z)$ - rather than in (9) - and this we do next.

Refer to Figure 1, and allow $\theta$ to increase through the interval

$$
\theta_{0} \leq \theta \leq \theta_{0}+2 \pi
$$

from its initial value $\theta_{0}$. The contour $C^{(n)}$ shifts clockwise, with its local orientation at $w^{(n)}$ turning through an angle of $\pi$. At first, $C^{(n)}$ deforms in a continuous manner, but as $\theta$ passes through $-\theta^{(n m)}$, the manner of approach to (or from) $\infty$ suddenly changes as the adjacent contour $C^{(m)}$ is entrapped. After $\theta$ has passed through $-\theta^{(n m)}$, a contribution from the integral over $C^{(m)}$ has to be included. If $C^{(m)}$ is entrapped by $C_{+}^{(n)}$, so that $w^{(m)}$ is a saddle on the right, then the contribution in question from $C^{(m)}$ will be $+Y^{(m)}(z)$. If $C^{(m)}$ is entrapped by $C_{-}^{(n)}$, so that $w^{(m)}$ is a saddle on the left, the contribution will be $-Y^{(m)}\left(z \mathrm{e}^{-2 \pi i}\right)$. (Recall how $Y^{(m)}(\eta)$ is specified, in the description leading to (7) and (8).) We find it convenient to re-label the saddle points on the right by $m_{+}$and those on the left by $m_{-}$.

Suppose then that $\theta$ is increased from its initial value $\theta_{0}$, within the interval (10), so that $M_{+}$and $M_{-}$adjacent saddles on the right and left, respectively, are entrapped. 
We find that (9) becomes

$$
\begin{aligned}
Y^{(n)}(z)= & \frac{\mathrm{e}^{-z p^{(n)}}}{z^{\frac{1}{2}}} \frac{1}{2 \pi i} \sum_{m} \frac{1}{\left[p^{(n m)}\right]^{\frac{1}{2}}} \int_{0}^{\infty} \frac{v^{-\frac{1}{2}} \exp \left(v p^{(n)} / p^{(n m)}\right) Y^{(m)}\left(v / p^{(n m)}\right)}{1-v / z p^{(n m)}} \mathrm{d} v \\
& +\sum_{m_{+}}^{M_{+}} Y^{\left(m_{+}\right)}(z)-\sum_{m_{-}}^{M_{-}} Y^{\left(m_{-}\right)}\left(z \mathrm{e}^{-2 \pi i}\right) .
\end{aligned}
$$

Had we decreased $\theta$ from its initial value, the adjacent saddle points would have been entrapped in a different order, and the sums corresponding to those in (11) would have been of the form

$$
+\sum_{m_{+}}^{M_{+}} Y^{\left(m_{+}\right)}\left(z \mathrm{e}^{2 \pi i}\right)-\sum_{m_{-}}^{M_{-}} Y^{\left(m_{-}\right)}(z)
$$

for some (usually different) $M_{+}, M_{-}$. Formulae of the form (11) hold good within the various sectors of the $z$-plane separated by Stokes lines. To give a complete description, we also should set out the representation in the exceptional cases when $z$ is on a Stokes line. Suppose that $\theta=-\theta^{\left(n m_{1}\right)}$ and $w^{\left(m_{1}\right)}$ is an adjacent saddle point on the right: we find that (9) becomes

$$
\begin{aligned}
Y^{(n)}(z)= & \frac{\mathrm{e}^{-z p^{(n)}}}{z^{\frac{1}{2}}} \frac{1}{2 \pi i} \frac{1}{\left[p^{\left(n m_{1}\right)}\right]^{\frac{1}{2}}} P \int_{0}^{\infty} \frac{v^{-\frac{1}{2}} \exp \left(v p^{(n)} / p^{\left(n m_{1}\right)}\right) Y^{\left(m_{1}\right)}\left(v / p^{\left(n m_{1}\right)}\right)}{1-v / z p^{\left(n m_{1}\right)}} \mathrm{d} v \\
& +\frac{\mathrm{e}^{-z p^{(n)}}}{z^{\frac{1}{2}}} \frac{1}{2 \pi i} \sum_{m \neq m_{1}} \frac{1}{\left[p^{(n m)}\right]^{\frac{1}{2}}} \int_{0}^{\infty} \frac{v^{-\frac{1}{2}} \exp \left(v p^{(n)} / p^{(n m)}\right) Y^{(m)}\left(v / p^{(n m)}\right)}{1-v / z p^{(n m)}} \mathrm{d} v \\
& +\frac{1}{2} Y^{\left(m_{1}\right)}(z)+\sum_{m_{+}}^{M_{+}} Y^{\left(m_{+}\right)}(z)-\sum_{m_{-}}^{M_{-}} Y^{\left(m_{-}\right)}\left(z \mathrm{e}^{-2 \pi i}\right)
\end{aligned}
$$

where $P \int_{0}^{\infty}$ denotes the Cauchy principal-value integral. An analogous representation holds if $w^{\left(m_{1}\right)}$ is an adjacent saddle point on the left: the last three terms in (13) would be replaced by

$$
-\frac{1}{2} Y^{\left(m_{1}\right)}(z)+\sum_{m_{+}}^{M_{+}} Y^{\left(m_{+}\right)}\left(z \mathrm{e}^{2 \pi i}\right)-\sum_{m_{-}}^{M_{-}} Y^{\left(m_{-}\right)}(z) .
$$

It may happen that saddles on the left and right are entrapped on the same Stokes line; in this event, there would be two principal-value integrals and two $\frac{1}{2} Y$ contributions in (13).

Berry and Howls ([4], p. 659) remark that if $\theta$ changes by $2 \pi$, the original path of steepest descents is recreated, but with the opposite orientation. The force of this remark in the context of (11) is that

$$
Y^{(n)}\left(z \mathrm{e}^{2 \pi i}\right)=-Y^{(n)}(z)+\sum_{m_{+}} Y^{\left(m_{+}\right)}\left(z \mathrm{e}^{2 \pi i}\right)-\sum_{m_{-}} Y^{\left(m_{-}\right)}(z)
$$

an analytic continuation formula for $Y^{(n)}(z)$. We remark that this formula describes an exact relationship between the analytic function defined by the steepest-descents integral through the saddle point $w^{(n)}$ and those through the adjacent saddle points $w^{(m)}$.

The continuation formula (15) sometimes can be derived alternatively as follows. In special cases, it may be possible, after making the changes of variable $t=-v / p^{(n m)}$ 
in (9), to rotate the paths of integration on to the positive $t$-axis. In this circumstance, the right-hand side of (9) is actually expressed as a single Stieltjes transform, and an application of the inverse Stieltjes transform (Widder [23], p. 340) yields an analytic continuation formula equivalent to (15), with $z$ replaced by $-z$ (see, for example, Boyd [7], equation (71)).

2.3. Asymptotic properties. As we have just noted, the integrals appearing in (9) or (6) above may be regarded as Stieltjes transforms, defined, for example, by Wong [24], p. 295. It is well-known from recent literature how certain asymptotic properties can be established from representations of Stieltjes transform type, such as (9) or (6) above (e.g., Boyd [7], [8]). We summarise:

(i) The terms in the formal asymptotic expansion (3) can be found by expanding the denominators of (9) or (6) in ascending powers of $v / z p^{(n m)}$. The coefficients are given by

$$
a_{r}=\frac{1}{2 \pi i} \sum_{m} \frac{1}{\left[p^{(n m)}\right]^{r+\frac{1}{2}}} \int_{0}^{\infty} v^{r-\frac{1}{2}} \exp \left(v p^{(n)} / p^{(n m)}\right) Y^{(m)}\left(v / p^{(n m)}\right) \mathrm{d} v .
$$

(ii) The remainder which results when the series (3) is truncated after $N$ terms,

$$
\frac{\mathrm{e}^{-z p^{(n)}}}{z^{N+\frac{1}{2}}} \frac{1}{2 \pi i} \sum_{m} \frac{1}{\left[p^{(n m)}\right]^{N+\frac{1}{2}}} \int_{0}^{\infty} \frac{v^{N-\frac{1}{2}} \exp \left(v p^{(n)} / p^{(n m)}\right) Y^{(m)}\left(v / p^{(n m)}\right)}{1-v / z p^{(n m)}} \mathrm{d} v,
$$

may be readily bounded provided each $Y^{(m)}\left(v / p^{(n m)}\right)$ can be suitably bounded for $v>0$ (Boyd [7]).

(iii) On substituting the asymptotic expansions corresponding to (3) for each $Y^{(m)}$, say

$$
Y^{(m)}(\eta) \sim \frac{\mathrm{e}^{-\eta p^{(m)}}}{\eta^{\frac{1}{2}}} \sum_{s=0}^{\infty} \frac{a_{s}^{(m)}}{\eta^{s}}
$$

into the integrand of (16), one finds formally that for large $r$,

$$
a_{r} \sim \frac{1}{2 \pi i} \sum_{m} \sum_{s=0}^{\infty} \frac{\Gamma(r-s)}{\left[p^{(n m)}\right]^{r-s}} a_{s}^{(m)} .
$$

In (18), that branch of $\eta^{\frac{1}{2}}$ is taken for which $\operatorname{ph}\left(\eta^{\frac{1}{2}}\right)=-\frac{1}{2} \theta^{(n m)}$ when $\operatorname{ph}(\eta)=-\theta^{(n m)}$. It should be remarked that (19) is indeed only a formal result: plainly, it cannot make sense actually to include those terms for which $s \geq r$. One must make the result (19) more rigorous by truncating the series and bounding the remainder as in Boyd [8], equation (3.38), or Boyd [9].

(iv) Suppose now that the series (3) is truncated optimally, at (or near) the least term of the expansion, which from (19) will be when $N \approx\left|z p^{(n \hat{m})}\right|$ where $\hat{m}$ denotes the adjacent saddle with the least value of $\left|p^{(n \hat{m})}\right|$. We shall show later (in $\S 3.1$ ) that for the integral representations that we consider in this paper, the adjacent saddles all have the same value of $p^{(m)}$, so that the reference to $\hat{m}$ is superfluous.

Let us suppose that $\theta$ is increased from its initial value $\theta_{0}$ with $\theta_{0}<\theta<\theta_{0}+2 \pi$. The Stokes lines are located at $\theta=-\theta^{(n m)}$ modulo $2 \pi$. If $w^{(m)}$ is an adjacent saddle point on the right, say $w^{\left(m_{+}\right)}$, then under our assumption about the range of $\theta$, we infer that the corresponding Stokes line is crossed when $\theta=-\theta^{\left(n m_{+}\right)}$; if it is on the left, say $w^{\left(m_{-}\right)}$, when $\theta=-\theta^{\left(n m_{-}\right)}+2 \pi$. The remainder after truncation itself may be expanded by substituting the asymptotic expansions (18) for each $Y^{(m)}$ into the 
integrand of (17). One thus finds formally that the remainder after $N$ terms may be expressed

$$
\sum_{m} \frac{\mathrm{e}^{-z p^{\left(m_{ \pm}\right)}}}{z^{\frac{1}{2}}} \sum_{s=0}^{\infty} \frac{a_{s}^{\left(m_{ \pm}\right)}}{z^{s}}\left[-i(-1)^{N-s} F_{N-s}\left(z p^{\left(n m_{ \pm}\right)} \mathrm{e}^{ \pm \pi i}\right)\right]
$$

where the functions $F_{N-s}$ are defined in the Appendix by (114), and where the upper and lower signs must be used consistently, depending on whether $w^{\left(m_{ \pm}\right)}$is on the right or the left respectively. (One anticipates that (20) is an asymptotic, rather than a convergent, series, with only small values of $s$ being taken into account.) Now when $N \approx\left|z p^{\left(n m_{ \pm}\right)}\right|, s$ is not large, and $z$ is near the Stokes line, the calculations given in the Appendix show that the factors in the square brackets in (20) are closely approximated by

$$
\frac{1}{2} \operatorname{erfc}\left(c^{\left(m_{ \pm}\right)} \sqrt{\frac{N}{2}}\right)
$$

respectively (equation (120)), where from (117),

$$
\frac{1}{2}\left[c^{\left(m_{ \pm}\right)}\right]^{2}=1-z p^{\left(n m_{ \pm}\right)} / N+\ln \left(z p^{\left(n m_{ \pm}\right)} / N\right) .
$$

Under the assumptions, we have made about $N, s$, and $z$, the Taylor expansion (118) for $c$ yields

$$
c^{\left(m_{+}\right)} \approx-\left(\theta+\theta^{\left(n m_{+}\right)}\right), \quad c^{\left(m_{-}\right)} \approx-\left(\theta+\theta^{\left(n m_{-}\right)}-2 \pi\right) .
$$

(The right-hand sides in (23) are small.) One readily infers that the values of the factors in the square brackets in (20) change swiftly but smoothly from nearly 0 to nearly +1 as $\theta$ passes through the Stokes line, 'switching on' the exponentially small contributions from the adjacent saddles (the Berry smoothing of Stokes discontinuities (Berry (1989))). We remark that this factor of +1 is consistent (as it ought to be) with the respective contributions from $Y^{\left(m_{ \pm}\right)}$in (11).

Now let us suppose that $\theta$ is decreased from its initial value $\theta_{0}$ with $\theta_{0}-2 \pi<\theta<\theta_{0}$. In place of $(20)$, one finds

$$
\sum_{m} \frac{\mathrm{e}^{-z p^{\left(m_{ \pm}\right)}}}{z^{\frac{1}{2}}} \sum_{s=0}^{\infty} \frac{a_{s}^{\left(m_{ \pm}\right)}}{z^{s}}\left[-i(-1)^{N-s} F_{N-s}\left(z p^{\left(n m_{ \pm}\right)} \mathrm{e}^{\mp \pi i}\right)\right]
$$

where again the upper and lower signs must be used consistently. The factors in the square brackets in (24) are approximated by

$$
-\frac{1}{2} \operatorname{erfc}\left(-c^{\left(m_{ \pm}\right)} \sqrt{\frac{N}{2}}\right)
$$

respectively, when $N \approx\left|z p^{\left(n m_{ \pm}\right)}\right|, s$ is not large, and $z$ is near the Stokes line. The Taylor expansion (118) for $c$ yields

$$
c^{\left(m_{+}\right)} \approx-\left(\theta+\theta^{\left(n m_{+}\right)}+2 \pi\right), \quad c^{\left(m_{-}\right)} \approx-\left(\theta+\theta^{\left(n m_{-}\right)}\right) .
$$

Again, we have a swift but smooth change, in this case from nearly 0 to nearly -1 as $\theta$ passes through the Stokes line. This is consistent with the respective contributions from $Y^{\left(m_{ \pm}\right)}$in (12). 


\section{Solutions of linear second-order differential equations}

We restrict attention to linear second-order differential equations with the property that any of their solutions may be represented by linear combinations of integrals of the general form (2):

$$
\int_{C} \mathrm{e}^{-z p(w)} q(w) \mathrm{d} w
$$

for some contour $C$ which begins and ends at infinity in the $w$-plane. We further assume, conversely, that any representation of the form (27) is a solution of the differential equation. For example, Airy and Bessel functions, considered in $\S 4$ and $\S 5$, enjoy representations of this kind. In $\S 3.1$ below, we discuss a special feature which must characterise such integral representations. Then, in $\S 3.2$ and $\S 3.3$, we shall discuss two sorts of solutions of the differential equation, each of which enjoy special integral representations.

3.1. Classification of saddle points. With the above assumptions, we may claim that the steepest-descents integral (2) through $w^{(n)}$ - for any saddle point $w^{(n)}$, and for any value of $\theta-$ is a solution of the differential equation. Its asymptotic behavior for large $z$ is evidently characterised by the factor $\exp \left(-z p^{(n)}\right)$, and, because there are exactly two linearly independent solutions, we infer that there must be exactly two values which $p^{(n)}$ may take as $n$ ranges over all the saddle points. Therefore, the saddle points may be grouped in two classes: membership of a class by $w^{(n)}$ is determined by which of the two possible values is actually taken by $p^{(n)}$. We shall refer to the two classes as the solution classes. It can be shown that for restricted values of $\theta$, the solution classes can be characterised as solution subspaces, and this we do next.

The analytic continuation of any solution which is represented by a steepest-descents integral for some value of $z$ continues to be represented by the steepest-descents integral so long as $z$ does not cross a Stokes line (see our discussion between (6) and (9)). Moreover, it is clear that the set of all solutions represented by steepest-descents integrals through a given saddle point is actually a subspace of dimension 1 . Therefore, for values of $z$ in a sector between neighbouring Stokes lines, each solution class corresponds directly to a particular solution subspace. Of course, in different sectors, the solution classes generally correspond to different solution subspaces.

Consider now the application of the Berry-Howls approach to the steepest-descents integral through $w^{(n)}$. Because, by definition, each adjacent saddle $w^{(m)}$ is reached from $w^{(n)}$ by a steepest-descents path for some value of $\theta$, we deduce that $p^{(m)} \neq p^{(n)}$ for any adjacent saddle, so that none of the adjacent saddles $w^{(m)}$ is in the same solution class as $w^{(n)}$; hence, each of the adjacent saddles belongs to the same solution class, and the value of $p^{(m)}$ is the same for each adjacent saddle. Moreover, because an adjacent saddle $w^{(m)}$ must be located on the path of steepest descents emanating from $w^{(n)}$ when $\theta=-\theta^{(n m)}$, and the values taken by $-\theta^{(n m)}$ over the adjacent saddles can differ only by multiples of $2 \pi$, we infer that for any given saddle $w^{(n)}$ either

(Case A) there is exactly one adjacent saddle, or

(Case B) there are exactly two adjacent saddles (cf. (7) and (8)).

In Case B, there is one adjacent saddle on each of the two halves of the same steepestdescents contour.

The earlier formulae (9), (11)-(13), and (15) now simplify. In Case B, we find that there are two values of $m-$ one each of $m_{+}$and $m_{-}$. In particular, (13) simplifies to 


$$
\begin{aligned}
Y^{(n)}(z)= & \frac{\mathrm{e}^{-z p^{(n)}}}{z^{\frac{1}{2}}} \frac{1}{2 \pi i} \sum_{m} \frac{1}{\left[p^{(n m)}\right]^{\frac{1}{2}}} P \int_{0}^{\infty} \frac{v^{-\frac{1}{2}} \exp \left(v p^{(n)} / p^{(n m)}\right) Y^{(m)}\left(v / p^{(n m)}\right)}{1-v / z p^{(n m)}} \mathrm{d} v \\
& +\frac{1}{2} Y^{\left(m_{+}\right)}(z)-\frac{1}{2} Y^{\left(m_{-}\right)}\left(z \mathrm{e}^{-2 \pi i}\right)
\end{aligned}
$$

when $\theta=-\theta^{\left(n m_{+}\right)}$. The modification to (28) when $\theta=-\theta^{\left(n m_{-}\right)}$is that the last two terms are replaced by

$$
+\frac{1}{2} Y^{\left(m_{+}\right)}\left(z \mathrm{e}^{2 \pi i}\right)-\frac{1}{2} Y^{\left(m_{-}\right)}(z) .
$$

(Further simplification is feasible in $(28)$, since $\left[p^{\left(n m_{-}\right)}\right]^{\frac{1}{2}}=-\left[p^{\left(n m_{+}\right)}\right]^{\frac{1}{2}}$.) The analytic continuation formula (15) simplifies to

$$
Y^{(n)}\left(z \mathrm{e}^{2 \pi i}\right)=-Y^{(n)}(z)+Y^{\left(m_{+}\right)}\left(z \mathrm{e}^{2 \pi i}\right)-Y^{\left(m_{-}\right)}(z) .
$$

The corresponding formulae in Case A are simpler still: there is only one value of $m$ - only one of $m_{+}$or $m_{-}$is present.

3.2. Numerically satisfactory pairs of solutions. Since the solutions of a linear second-order linear differential equation form a vector space of dimension 2 , one can, of course, choose any pair of linearly independent solutions as a basis; that is to say, one can express any solution of the differential equation as a linear combination of the chosen pair. However, in the asymptotic limit with which we are concerned, $|z| \rightarrow \infty$ in the complex plane, it is well-known that linear independence of itself is inadequate for this purpose in both numerical and asymptotic computations (Olver [19], p. 154). Instead, one needs to span the space with a pair of solutions which enjoy a stronger property: that of being numerically satisfactory (the term is due to Miller - see Olver [19], p. 154). The essential idea is that, over a range of $\theta$, one should choose the spanning pair so that (except at isolated values of $z$ ) severe cancellation cannot take place between the terms in any linear combination.

We shall show that pairs of numerically satisfactory solutions arise naturally from integral representations of the form (27). Before doing so, we remark on the significance of Stokes lines and anti-Stokes lines in this context.

For the saddle point $w^{(n)}$ of an integral representation of the form (27), the Stokes lines are the directions for which $\theta=-\theta^{(n m)}$. As $z$ varies, it is on Stokes lines that the contributions from the adjacent saddles first appear. Just after $z$ passes through a Stokes line, the contribution from the adjacent saddle $w^{(m)}$ will be exponentially small compared with that from $w^{(n)}$. However, as $z$ varies further, the relative contributions from the two saddles become more comparable, until $\theta=-\theta^{(n m)} \pm \pi / 2$, when the two contributions are of the same order of magnitude. The values of $z$ for which $\theta=$ $-\theta^{(n m)} \pm \pi / 2$ are anti-Stokes lines. Just after $z$ passes through the anti-Stokes line, the contribution from the adjacent saddle $w^{(m)}$ will be exponentially large compared with that from $w^{(n)}$.

Consider two mutually adjacent saddle points $w^{(n)}$ and $w^{(m)}$, so that one is in each of the solution classes. Recall (refer to our discussion just above equations (7) and (8)) how the steepest-descents integrals $Y^{(n)}(z)$ and $Y^{(m)}(z)$ are specified. The first of these, $Y^{(n)}(z)$, is defined initially at $\theta=\theta_{0}$, and elsewhere by analytic continuation. The second, $Y^{(m)}(z)$, is defined initially at $\theta=-\theta^{(n m)}$ (where $-\theta^{(n m)}$ satisfies either (7) or (8) depending on whether $w^{(m)}$ is adjacent to $w^{(n)}$ on the right or the left), 
and is defined elsewhere by analytic continuation. To be definite, let us suppose that $w^{(m)}$ is adjacent on the left (this will be convenient for our example in $\S 4$ ). We thus have defined solutions $Y^{(n)}(z)$ and $Y^{(m)}(z)$ which are the steepest-descents integrals through the saddles $w^{(n)}$ and $w^{(m)}$ when $-\theta^{(n m)}<\theta<2 \pi-\theta^{(n m)}$ and $-\pi-\theta^{(n m)}<\theta<\pi-\theta^{(n m)}$, respectively, and are defined for other values of $\theta$ by analytic continuation. Except for a slowly-varying factor, the asymptotic behavior of $Y^{(n)}(z)$ is given by

$$
\mathrm{e}^{-z p^{(n)}} \quad \text { as }|z| \rightarrow \infty, \quad-\pi / 2-\theta^{(n m)}<\theta<5 \pi / 2-\theta^{(n m)}
$$

likewise, the asymptotic behavior of $Y^{(m)}(z)$ is given by

$$
\mathrm{e}^{-z p^{(m)}} \quad \text { as }|z| \rightarrow \infty, \quad-3 \pi / 2-\theta^{(n m)}<\theta<3 \pi / 2-\theta^{(n m)} .
$$

It is evident that $\left\{Y^{(n)}(z), Y^{(m)}(z)\right\}$ constitutes a numerically satisfactory pair in $-\pi / 2-\theta^{(n m)}<\theta<3 \pi / 2-\theta^{(n m)}$ (the sector boundaries are anti-Stokes lines). If $w^{(m)}$ had been adjacent on the right, we should have found a numerically satisfactory pair in the sector $-3 \pi / 2-\theta^{(n m)}<\theta<\pi / 2-\theta^{(n m)}$.

We have already, in $\S 2.3$, discussed how the asymptotic properties of solutions such as $Y^{(n)}(z)$ and $Y^{(m)}(z)$ may be established.

It is easy to show that such pairs of numerically satisfactory solutions can be specified in any sector of angle $2 \pi$ bounded by anti-Stokes lines. See, for example, our comments in the paragraph following (57).

3.3. Dominant solutions of Bi type associated with a Stokes line. We continue to assume that the steepest-descents integral $Y^{(n)}(w)$ through the saddle point $w^{(n)}$ is defined at $\theta=\theta_{0}$. Let us suppose, to be definite, that there is a saddle point adjacent on the right, $w^{\left(m_{+}\right)}$, and consider $z$ varying near the Stokes line $\theta=-\theta^{\left(n m_{+}\right)}$ (this will be convenient for our example in $\S 4$ ). The steepest-descents integrals through $w^{(n)}$ correspond to different analytic functions, depending on whether the steepest-descents paths pass the adjacent saddle $w^{\left(m_{+}\right)}$on the left or the right, that is, depending on whether $\theta$ is less or greater than $-\theta^{\left(n m_{+}\right)}$. These two analytic functions have the same (Poincaré) asymptotic expansion: their difference is exponentially small (compared with the asymptotic behavior of the functions themselves). In both cases the functions are dominant solutions of the differential equation. Now, the set of all dominant solutions comprises almost the whole of the solution space of the differential equation - only the one-dimensional subspace of recessive solutions is excluded. Clearly, therefore, the property of dominance of itself cannot be used to define a standard solution of the differential equation. If one wishes to specify a dominant solution, appeal must be made to a further distinctive attribute.

One possibility is to define a dominant solution as the analytic continuation of a recessive solution (as we effectively did in $\S 3.2$ ). There have been attempts to define dominant solutions uniquely by means other than this. See, for example, the comments by Dingle ([14], p. 17, or p. 293-295). In the past, such approaches have implicitly relied on an additional attribute, such as interpretive rules (Dingle), or choice of contour path when inverting a Borel transform. Very recently, Costin and Kruskal [12] introduced the notion of the solution of a differential equation being optimally asymptotic to formal asymptotic series, and showed how, for each value of $\theta$, solutions and formal series could be identified uniquely with each other; we discuss the connection between their work and ours in $\S 6$. We shall proceed differently: we propose a definition based on specific combinations of steepest-descents contours. We shall find that such a definition yields specific asymptotic behavior at optimal 
truncation, and in $\S 6$, we show how this behavior itself naturally suggests a definition which can be applied to a wide class of asymptotic expansions.

Dominant solutions are at their most dominant on a Stokes line: in particular, for the situation described in the previous paragraph, on the line $\theta=-\theta^{\left(n m_{+}\right)}$. Suppose that we wish to define a dominant solution in terms of its properties near this Stokes line, and do so in terms of steepest-descents integrals through the saddle $w^{(n)}$. Let us consider the two steepest-descents integrals

$$
\begin{aligned}
& J_{-}=\int_{C^{(n)}\left(-\theta^{\left(n m_{+}\right)}-\right)} \mathrm{e}^{-z p(w)} q(w) \mathrm{d} w \\
& J_{+}=\int_{C^{(n)}\left(-\theta^{\left(n m_{+}\right)}+\right)} \mathrm{e}^{-z p(w)} q(w) \mathrm{d} w
\end{aligned}
$$

where the contours $C^{(n)}\left(-\theta^{\left(n m_{+}\right)}-\right)$and $C^{(n)}\left(-\theta^{\left(n m_{+}\right)}+\right)$denote the "dogleg" paths of steepest descents through $w^{(n)}$ which turn left and right, respectively, at the saddle $w^{\left(m_{+}\right)}$, and which are the limits of the steepest-descents contours as $\theta \rightarrow-\theta^{\left(n m_{+}\right)}$from below and from above for $\theta<-\theta^{\left(n m_{+}\right)}$and $\theta>-\theta^{\left(n m_{+}\right)}$, respectively (see Figure 2). Both $J_{-}$and $J_{+}$are dominant solutions when $\theta=-\theta^{\left(n m_{+}\right)}$. More generally, almost all linear combinations of these two steepest-descents integrals yield dominant solutions. However, to within a multiplicative constant, there is only one combination which is symmetric with respect to each side of the Stokes line and which yields a dominant solution: the function, which we denote by $y^{(n)}(z)$, which is defined as the sum of the two steepest-descents integrals,

$$
y^{(n)}(z)=J_{-}+J_{+},
$$

when $\theta=-\theta^{\left(n m_{+}\right)}$.

We thus are led to define (compare the definition (2) for $Y^{(n)}(z)$ )

$$
y^{(n)}(z)=\left\{\int_{C^{(n)}\left(-\theta^{\left(n m_{+}\right)}-\right)}+\int_{C^{(n)}\left(-\theta^{\left(n m_{+}\right)}+\right)}\right\} \mathrm{e}^{-z p(w)} q(w) \mathrm{d} w
$$

for values of $z$ on the Stokes line $\theta=-\theta^{\left(n m_{+}\right)}$, and elsewhere by analytic continuation. Effectively then, the further attribute we have used to specify a particular dominant solution is a certain symmetry condition with respect to the Stokes line. The simplest example of such a function is a solution to Airy's equation: $\mathrm{Bi}(z)$, which is associated with the Stokes line comprising real positive $z$. For this reason, we refer to such a solution as the solution of $\mathrm{Bi}$ type associated with a Stokes line.

We remark that the definition (35) can, of course, only apply in Case A if there actually is a saddle point on the right! In Case B, (35) always is well-defined, though it should be noted that the definition is not symmetric between $w^{\left(m_{+}\right)}$and $w^{\left(m_{-}\right)}$. Our discussion below is for Case B. The modifications required for Case A are trivial: merely removing references to $w^{\left(m_{-}\right)}$in the various formulae.

A representation, analogous to earlier representations of Stieltjes transform type, may be found for the solution $y^{(n)}(z)$ from previous results. First, we note that when $\theta=-\theta^{\left(n m_{+}\right)}$,

$$
J_{-}-J_{+}=Y^{\left(m_{+}\right)}(z)-Y^{\left(m_{-}\right)}\left(z \mathrm{e}^{-2 \pi i}\right),
$$

and so we may eliminate $J_{+}$between (34) and (36) to yield an expression for $y^{(n)}$ in terms of $J_{-}$. Then, we note that the left-hand side of (28) is actually $J_{-}$, and so we 


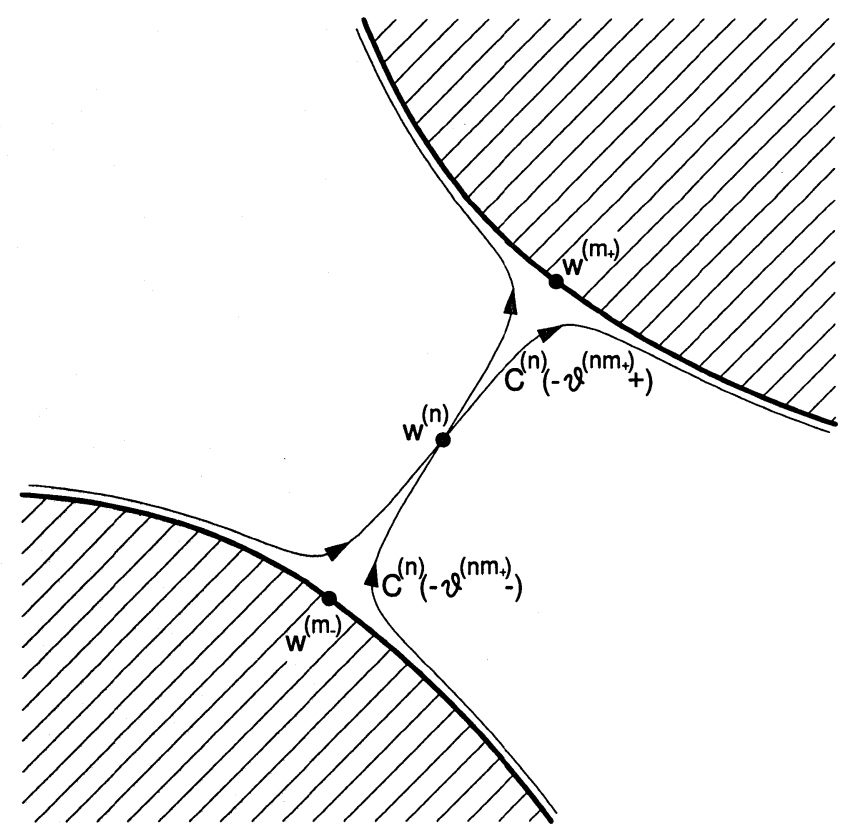

FIgure 2. A depiction of the "dogleg" contours $C^{(n)}\left(-\theta^{\left(n m_{+}\right)}-\right)$and $C^{(n)}\left(-\theta^{\left(n m_{+}\right)}+\right)$, steepest-descents paths through $w^{(n)}$ which respectively turn left and right at the saddle $w^{\left(m_{+}\right)}$, and which are the limits of the steepest-descents contours as $\theta \rightarrow-\theta^{\left(n m_{+}\right)}$from below and from above. (For the purposes of illustration, the contours have actually been drawn for values of $\theta$ close to, but different from, $\theta^{\left(n m_{+}\right)}$.)

establish the representation

$$
y^{(n)}(z)=\frac{\mathrm{e}^{-z p^{(n)}}}{z^{\frac{1}{2}}} \frac{1}{\pi i} \sum_{m} \frac{1}{\left[p^{(n m)}\right]^{\frac{1}{2}}} P \int_{0}^{\infty} \frac{v^{-\frac{1}{2}} \exp \left(v p^{(n)} / p^{(n m)}\right) Y^{(m)}\left(v / p^{(n m)}\right)}{1-v / z p^{(n m)}} \mathrm{d} v
$$

on the Stokes line $\theta=-\theta^{\left(n m_{+}\right)}$; for other values of $\theta$, analytic continuation may be used. The integrals in (37) are properly described, not as of Stieltjes transform type, but as one-sided Hilbert transforms (Wong [24], p. 313).

Recall that the definition (35) for $y^{(n)}(z)$, and its representation (37) of Hilbert transform type, have been given under the assumption (made at the beginning of the subsection) that $w^{\left(m_{+}\right)}$is an adjacent saddle point on the right. If $w^{\left(m_{-}\right)}$were an adjacent saddle point on the left, the corresponding definition would, of course, still be feasible, but the right-hand side of (37) would then represent, not $y^{(n)}(z)$, but $-y^{(n)}(z)$. 
Analytic continuation formulae are easily established from (37). Thus, when $z$ is in the sector $-\theta^{\left(n m_{+}\right)}<\theta<2 \pi-\theta^{\left(n m_{+}\right)}$, we find (cf. (11))

$$
\begin{gathered}
y^{(n)}(z)=\frac{\mathrm{e}^{-z p^{(n)}}}{z^{\frac{1}{2}}} \frac{1}{\pi i} \sum_{m} \frac{1}{\left[p^{(n m)}\right]^{\frac{1}{2}}} \int_{0}^{\infty} \frac{v^{-\frac{1}{2}} \exp \left(v p^{(n)} / p^{(n m)}\right) Y^{(m)}\left(v / p^{(n m)}\right)}{1-v / z p^{(n m)}} \mathrm{d} v \\
+Y^{\left(m_{+}\right)}(z)-Y^{\left(m_{-}\right)}\left(z \mathrm{e}^{-2 \pi i}\right),
\end{gathered}
$$

while if $z$ were in the sector $-2 \pi-\theta^{\left(n m_{+}\right)}<\theta<-\theta^{\left(n m_{+}\right)}$, the latter two terms would be replaced by (cf. (12))

$$
-Y^{\left(m_{+}\right)}(z)+Y^{\left(m_{-}\right)}\left(z \mathrm{e}^{-2 \pi i}\right) .
$$

The analogue of the analytic continuation formula (30) for $Y^{(n)}$ is, from (38) and (39),

$$
\begin{aligned}
y^{(n)}\left(z \mathrm{e}^{2 \pi i}\right)=- & y^{(n)}(z)+Y^{\left(m_{+}\right)}\left(z \mathrm{e}^{2 \pi i}\right) \\
& -Y^{\left(m_{+}\right)}(z)-Y^{\left(m_{-}\right)}(z)+Y^{\left(m_{-}\right)}\left(z \mathrm{e}^{-2 \pi i}\right) .
\end{aligned}
$$

Expanding the integrand of (37) formally in inverse powers of $z$ yields the asymptotic expansion

$$
y^{(n)}(z) \sim 2 \frac{\mathrm{e}^{-z p^{(n)}}}{z^{\frac{1}{2}}} \sum_{r=0}^{\infty} \frac{a_{r}}{z^{r}} .
$$

We shall show in (ii) below that this expansion is valid for $|z| \rightarrow \infty$ in the sector $-\pi / 2-\theta^{\left(n m_{+}\right)}<\theta<\pi / 2-\theta^{\left(n m_{+}\right)}$. Much of the analysis of the integral representations of Stieltjes transform type summarised in $\S 2.3$ carries over to the representation of Hilbert transform type given in (37) above. We consider in turn the properties (i)-(iv) listed in $\S 2.3$.

(i) Equation (16) for the coefficient $a_{r}$ holds good, as in $\S 2.3$.

(ii) Equation (17) for the remainder holds good for $\theta=-\theta^{\left(n m_{+}\right)}$(with $2 \pi i$ replaced by $\pi i$, and with the Cauchy principal value understood). However, because of the cancellation implicit in taking Cauchy principal values, the remainder cannot be realistically bounded in the direct way that (17) can. For other values of $\theta$, the representations (38) and (39) need to be invoked, so that we have to bound integrals of Stieltjes transform type. This can be accomplished, as we noted in $\S 2.3$. In particular, one can show that (41) is indeed an asymptotic expansion in the Poincaré sense for $|z| \rightarrow \infty$ in the sector $-\pi / 2-\theta^{\left(n m_{+}\right)}<\theta<\pi / 2-\theta^{\left(n m_{+}\right)}$. On the anti-Stokes lines $\pm \pi / 2-\theta^{\left(n m_{+}\right)}$, the contributions from the $Y^{\left(m_{ \pm}\right)}$terms in (38) and (39) become comparable with that from the integral in (38).

(iii) The asymptotic estimate (19) for the coefficient $a_{r}$ holds good, as in $\S 2.3$.

(iv) The remainder when the series in (41) is truncated after $N$ terms is found formally to be

$$
\sum_{m} \frac{\mathrm{e}^{-z p^{\left(m_{ \pm}\right)}}}{z^{\frac{1}{2}}} \sum_{s=0}^{\infty} \frac{a_{s}^{\left(m_{ \pm}\right)}}{z^{s}}\left[2 i \bar{F}_{N-s}\left(z p^{\left(n m_{ \pm}\right)} \mathrm{e}^{i \phi_{ \pm}}\right)\right]
$$

where the functions $\bar{F}_{N-s}$ are defined in the Appendix by (127), where the upper and lower signs must be used consistently, and where $\phi_{+}=0$ and $\phi_{-}=-2 \pi$. When $N \approx\left|z p^{\left(n m_{ \pm}\right)}\right|, s$ is not large, and $z$ is near the Stokes line $\theta=-\theta^{\left(n m_{+}\right)}$, the factors 
in the square brackets in (42) are closely approximated by

$$
-\operatorname{erf}\left(c^{\left(m_{+}\right)} \sqrt{\frac{N}{2}}\right)
$$

respectively (see (129)), where $c^{\left(m_{+}\right)}$is defined in (22). In this case (cf. (23) and (26)),

$$
c^{\left(m_{+}\right)} \approx-\left(\theta+\theta^{\left(n m_{+}\right)}\right) \text {. }
$$

We infer that as $\theta$ passes through the Stokes line, the factors in the square brackets in (42) take values which change rapidly from nearly 0 on the Stokes line itself to nearly $+1\left(\theta>-\theta^{\left(n m_{+}\right)}\right)$and to nearly $-1\left(\theta<-\theta^{\left(n m_{+}\right)}\right)$. These factors of +1 and -1 are consistent with the contributions from $Y^{\left(m_{ \pm}\right)}$in (38) and (39), respectively.

The formal similarity between (20) and (42) should be noted: the square brackets have behavior of erfc and erf type, respectively, near the Stokes line $\theta=-\theta^{\left(n m_{+}\right)}$. The error function behavior in (42) has the effect of rapidly but smoothly switching the signs of the exponentially small contributions of the adjacent saddles as the Stokes line is crossed. As we note at the end of the Appendix, it was just this behavior which Berry [3] addressed in his original work on the Stokes phenomenon.

We have shown that solutions of Bi type associated with a Stokes line enjoy the specific error function behavior (43) near the Stokes line. Our definition of "the solution of Bi type associated with a Stokes line", in (35), invokes a special combination of steepest-descents contours. It is natural to speculate that the specific error function behavior (43) might occur more generally, as for example when integral representations of solutions are not available. We address this question in $\S 6$.

\section{Airy functions}

In this section, we illustrate our earlier, general discussions by reference to Airy's differential equation,

$$
\frac{\mathrm{d}^{2} W}{\mathrm{~d} Z^{2}}-Z W=0
$$

Standard solutions of the equation are the Airy functions $\operatorname{Ai}(Z)$ and $\operatorname{Bi}(Z)$, which enjoy the integral representations

$$
\operatorname{Ai}(Z)=\frac{1}{2 \pi i} \int_{\infty e^{-i \pi / 3}}^{\infty e^{i \pi / 3}} e^{\frac{1}{3} t^{3}-Z t} \mathrm{~d} t
$$

and

$$
\operatorname{Bi}(Z)=\frac{1}{2 \pi}\left\{\int_{\infty e^{i \pi / 3}}^{-\infty}+\int_{-\infty}^{\infty \mathrm{e}^{-i \pi / 3}}\right\} e^{\frac{1}{3} t^{3}-Z t} \mathrm{~d} t
$$

for complex $Z$ (see, for example, Jeffreys [17], § 2.5, or Wong [24], p. 90).

For our purposes, it is more convenient to consider Airy functions of argument $\left(\frac{3}{2} z\right)^{\frac{2}{3}}$,as in Boyd [7], p. 507. Thus

$$
2 \pi i\left(\frac{3}{2} z\right)^{-\frac{1}{3}} \operatorname{Ai}\left(\left(\frac{3}{2} z\right)^{\frac{2}{3}}\right)=\int_{C_{1}} \mathrm{e}^{-z p(w)} \mathrm{d} w
$$

and

$$
2 \pi\left(\frac{3}{2} z\right)^{-\frac{1}{3}} \operatorname{Bi}\left(\left(\frac{3}{2} z\right)^{\frac{2}{3}}\right)=\left\{\int_{C_{2}}+\int_{C_{3}}\right\} \mathrm{e}^{-z p(w)} \mathrm{d} w
$$


where $p(w)$ is defined (for this section only) as

$$
p(w)=-\frac{1}{2} w^{3}+\frac{3}{2} w .
$$

In the representations (48) and (49), the contours $C_{1}, C_{2}, C_{3}$ in the $w$-plane are the same, for $z>0$, as those in (46) and (47) in the $t$-plane; the representations (48) and (49) may be extended to other values of $z$ by analytic continuation. The function $p(w)$ defined in (50) has two saddle points, at $w^{(1)}=1$ and $w^{(-1)}=-1$.

Now let us consider the solutions of Airy's equation (45) in the context of our discussions in $\S 2$ and $\S 3$. Thus, after the transformations

$$
z=\frac{2}{3} Z^{\frac{3}{2}} \quad \text { and } \quad Y(z)=Z^{-\frac{1}{2}} W(Z)
$$

of the independent and dependent variables, the differential equation (45) for $W(Z)$ is transformed to the second-order linear differential equation

$$
z^{2} \frac{\mathrm{d}^{2} Y}{\mathrm{~d} z^{2}}+z \frac{\mathrm{d} Y}{\mathrm{~d} z}-\left(z^{2}+\frac{1}{9}\right) Y=0
$$

(actually the modified Bessel equation with $\nu=\frac{1}{3}$, cf. (77)). Evidently then, the integral representations on the right-hand sides of (48) and (49) are solutions of (52); moreover, any integral of the integrand in (48) and (49) whose contour begins and ends at infinity is a solution of (52). Thus, our discussions in $\S 3$ (and $\S 2$ ) apply directly, provided the conditions on $p(w)$ referred to in the paragraph between (3) and (4) hold good. The conditions indeed do hold (Boyd [7], p. 507).

We showed in $\S 3.1$ that, quite generally, the various saddle points divide into two solution classes. In the present example, there are in fact only two saddle points, $w^{(1)}=1$ and $w^{(-1)}=-1$, which are plainly in different solution classes. We also showed in $\S 3.1$ that in each sector bounded by neighbouring Stokes lines, the solution classes correspond to particular solution subspaces. For this problem, the Stokes lines are located at $\theta=0, \pm \pi, \pm 2 \pi, \ldots$ In, for example, the sector $-\pi<\theta<0$ (or equivalently $-2 \pi / 3<\operatorname{ph}(Z)<0$ ), we infer from the calculations in the next paragraph that the particular subspaces comprise solutions which are multiples of $\operatorname{Ai}(Z)$ and of $\operatorname{Ai}\left(Z \mathrm{e}^{2 \pi i / 3}\right)$.

To apply the result about numerically satisfactory solutions presented in $\S 3.2$, let $n=1, \theta_{0}=0$, and specify the orientation of the contour $C^{(1)}\left(\theta_{0}\right)$ by requiring that it be traversed upwards. Then $m=-1$, and $-\theta^{(n m)}=-\pi$. We consider the solution $Y^{(1)}(z)$, defined to be the integral

$$
Y^{(1)}(z)=\int_{C^{(1)}(\theta)} \mathrm{e}^{-z p(w)} \mathrm{d} t
$$

when $\theta=\theta_{0}=0$ (where $C^{(1)}(0)$ is traversed upwards), and the solution $Y^{(-1)}(z)$, defined to be the integral

$$
Y^{(-1)}(z)=\int_{C^{(-1)}(\theta)} \mathrm{e}^{-z p(w)} \mathrm{d} t
$$

when $\theta=-\theta^{(n m)}=-\pi$ (where $C^{(-1)}(-\pi)$ is traversed downwards). For other values of $\theta$, the solutions $Y^{(1)}(z)$ and $Y^{(-1)}(z)$ are defined by analytic continuation. In particular, $Y^{(1)}(z)$ is represented by the formula (53) for $-\pi<\theta<\pi$, and $Y^{(-1)}(z)$ is represented by (54) for $-2 \pi<\theta<0$. Except for a slowly-varying factor, the 


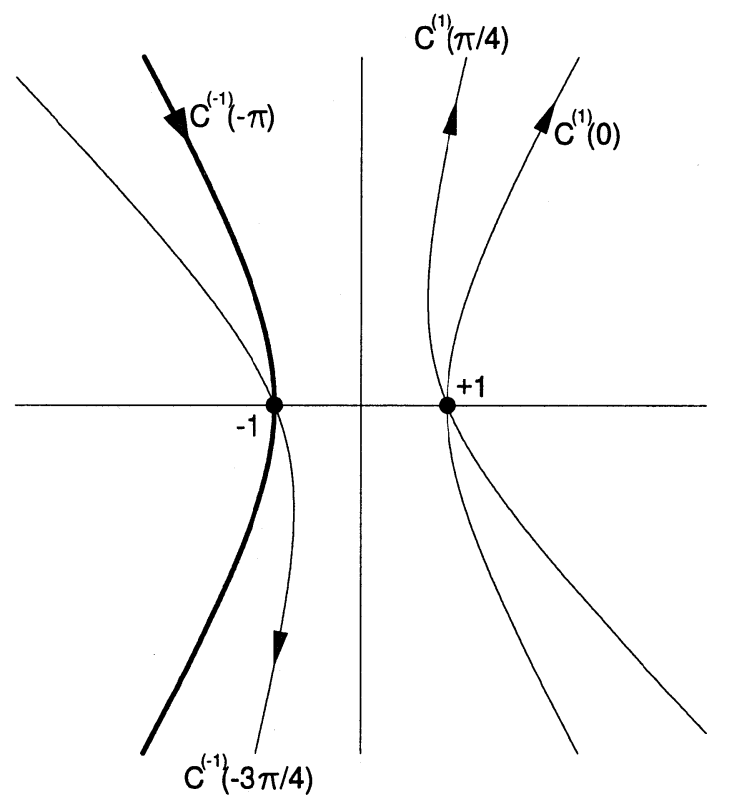

FiguRE 3. The contours $C^{(1)}(0)$ and $C^{(-1)}(-\pi)$, specified in (53) and (54). Also shown are the contours $C^{(1)}(\pi / 4)$ and $C^{(-1)}(-3 \pi / 4)$. (The contour $C^{(-1)}(\theta)$ is re-defined above (69).)

asymptotic behavior of $Y^{(1)}(z)$ is given by

$$
e^{-z} \quad \text { as }|z| \rightarrow \infty, \quad-3 \pi / 2<\theta<3 \pi / 2,
$$

whilst that for $Y^{(-1)}(z)$ is given by

$$
\mathrm{e}^{z} \quad \text { as }|z| \rightarrow \infty, \quad-5 \pi / 2<\theta<\pi / 2 .
$$

It is evident that $\left\{Y^{(-1)}(z), Y^{(1)}(z)\right\}$ constitutes a numerically satisfactory pair in $-3 \pi / 2<\theta<\pi / 2$. One readily identifies the solutions $Y^{(1)}(z)$ and $Y^{(-1)}(z)$ of the differential equation (52) with solutions of Airy's equation (45):

$$
\begin{aligned}
Y^{(1)}\left(\frac{2}{3} Z^{\frac{3}{2}}\right) & =2 \pi i Z^{-\frac{1}{2}} \mathrm{Ai}(Z), \\
Y^{(-1)}\left(\frac{2}{3} Z^{\frac{3}{2}}\right) & =-2 \pi i Z^{-\frac{1}{2}} \mathrm{e}^{\frac{2}{3} \pi i} \mathrm{Ai}\left(Z^{\frac{2}{3} \pi i}\right),
\end{aligned}
$$

for $-\pi<\operatorname{ph}(Z)<\pi / 3$, and elsewhere by analytic continuation. It is, of course, wellknown that $\left\{\operatorname{Ai}(Z), \operatorname{Ai}\left(Z \mathrm{e}^{\frac{2}{3} \pi i}\right)\right\}$ is a numerically satisfactory pair in $-\pi<\operatorname{ph}(Z)<$ $\pi / 3$ (Olver [19], p. 414).

One can define numerically satisfactory pairs of solutions in other sectors bounded by anti-Stokes lines by different choices of $\theta_{0}$ or different orientations of $C^{(1)}\left(\theta_{0}\right)$ (e.g., for $-\pi / 2<\theta<3 \pi / 2$, choose $\theta_{0}=0$, but take $C^{(1)}\left(\theta_{0}\right)$ downwards). 
The asymptotic properties of $Y^{(1)}(z)$ and $Y^{(-1)}(z)$ may be found from their integral representations (9) of Stieltjes transform type. That for $Y^{(1)}(z)$ is

$$
Y^{(1)}(z)=-\frac{1}{2 \pi} \frac{e^{-z}}{z^{\frac{1}{2}}} \int_{0}^{\infty} \frac{t^{-\frac{1}{2}} \mathrm{e}^{-t} Y^{(-1)}\left(t \mathrm{e}^{-\pi i}\right)}{1+t / z} \mathrm{~d} t
$$

valid for $-\pi<\theta<\pi$. In the present problem, it can be shown that $Y^{(1)}$ and $Y^{(-1)}$ may be expressed in terms of each other, though of course remaining linearly independent, and this yields considerable simplification. The identity

$$
Y^{(-1)}(z)=-Y^{(1)}\left(z \mathrm{e}^{\pi i}\right)
$$

(cf. Boyd [7], equation (55)) shows that formula (58) alternatively may be written

$$
Y^{(1)}(z)=\frac{1}{2 \pi} \frac{e^{-z}}{z^{\frac{1}{2}}} \int_{0}^{\infty} \frac{t^{-\frac{1}{2}} \mathrm{e}^{-t} Y^{(1)}(t)}{1+t / z} \mathrm{~d} t
$$

and also that to discuss the properties of $Y^{(-1)}(z)$, it suffices to consider $Y^{(1)}(z)$. The various asymptotic properties for $Y^{(1)}(z)$ can be established along the lines discussed in $\S 2.3$. The asymptotic expansion is in the form

$$
Y^{(1)}(z) \sim \frac{\mathrm{e}^{-z}}{z^{\frac{1}{2}}} i \sum_{r=0}^{\infty} \frac{(-1)^{r} b_{r}}{z^{r}}
$$

as $|z| \rightarrow \infty$ in $-3 \pi / 2<\theta<3 \pi / 2$ where the coefficients $b_{r}$, all of which are positive, are given by

$$
b_{r}=-\frac{\mathrm{i}}{2 \pi} \int_{0}^{\infty} t^{r-\frac{1}{2}} e^{-t} Y^{(1)}(t) \mathrm{d} t=\sqrt{\frac{2 \pi}{3}} \frac{\Gamma\left(3 r+\frac{1}{2}\right)}{54^{r} r ! \Gamma\left(r+\frac{1}{2}\right)} .
$$

This latter result is given in equation (50) of Boyd [7], $\S 5$, where it also is shown how the remainder term (cf. (17)) may be bounded. For large $r$, we find that the coefficients are approximated by (cf. (19))

$$
b_{r} \sim \frac{1}{2 \pi} \sum_{s=0}^{\infty}(-1)^{s} \frac{\Gamma(r-s)}{2^{r-s}} b_{s} .
$$

The remainder after optimal truncation in (61), say after $N$ terms, may, as in (24) and (25), be approximated by

$$
-\frac{\mathrm{e}^{z}}{z^{\frac{1}{2}}} \sum_{s=0} \frac{b_{s}}{z^{s}}\left[-\frac{1}{2} \operatorname{erfc}\left(-c^{(-1)} \sqrt{\frac{N}{2}}\right)\right]
$$

for large $|z|, \theta$ near $-\pi$, and where $c^{(-1)}$ is given by (22) with $p^{(1,-1)}=-2$. (We use the notation $\sum_{s=0}$ to denote that $s$ is restricted to small values.) In this problem, the approximation $(26)$ is

$$
c^{(-1)} \approx-(\theta+\pi) .
$$

With account taken of the asymptotic expansion for $Y^{(-1)}(z)$,

$$
Y^{(-1)}(z) \sim-\frac{\mathrm{e}^{z}}{z^{\frac{1}{2}}} \sum_{s=0}^{\infty} \frac{b_{s}}{z^{s}}
$$

when $-5 \pi / 2<\theta<\pi / 2$, we find, as expected, that (64) exhibits the Berry smoothing of the Stokes discontinuity across the Stokes line $\theta=-\pi$ of the kind discussed below (26). The corresponding behavior of $Y^{(1)}(z)$ for $\theta$ near $+\pi$ likewise could be established. 
The continuation formula (30) for $Y^{(n)}(z)$ is, in this case,

$$
Y^{(1)}\left(z \mathrm{e}^{2 \pi i}\right)=-Y^{(1)}(z)-Y^{(-1)}(z)
$$

From (57) and (59), this is equivalent to

$$
\mathrm{e}^{-\frac{2}{3} \pi i} \operatorname{Ai}\left(Z \mathrm{e}^{-\frac{2}{3} \pi i}\right)+\operatorname{Ai}(Z)+\mathrm{e}^{\frac{2}{3} \pi i} \operatorname{Ai}\left(Z \mathrm{e}^{\frac{2}{3} \pi i}\right)=0
$$

the well-known connection formula for Airy functions (Abramowitz and Stegun [1], equation (10.4.7)).

In $\S 3.3$, we introduced $y^{(n)}(z)$, "the solution of Bi type associated with a Stokes line". Not surprisingly, in the present example, this will turn out to correspond to Bi itself! To apply the results of $\S 3.3$, we take $n=-1, \theta_{0}=-\pi$, and specify the orientation of the contour $C^{(-1)}\left(\theta_{0}\right)$ by requiring that it be traversed upwards (in contrast with our earlier definition below (54)). In the notation of $\S 3.3, m_{+}=1$ and $\theta^{\left(n m_{+}\right)}=0$. The definition of $(35)$ for $y^{(-1)}(z)$ is

$$
y^{(-1)}(z)=\left\{\int_{C^{(-1)}(0-)}+\int_{C^{(-1)}(0+)}\right\} e^{-z p(w)} \mathrm{d} w
$$

when $\theta=0$; elsewhere, $y^{(-1)}(z)$ is defined by analytic continuation. From (49), it is evident that $y^{(-1)}(z)$ corresponds to $\operatorname{Bi}(Z)$ :

$$
y^{(-1)}\left(\frac{2}{3} Z^{\frac{3}{2}}\right)=2 \pi Z^{-\frac{1}{2}} \operatorname{Bi}(Z) .
$$

The integral representation of Hilbert transform type for $y^{(-1)}(z)$ given by (37) is

$$
y^{(-1)}(z)=\frac{1}{\pi i} \frac{\mathrm{e}^{z}}{z^{\frac{1}{2}}} P \int_{0}^{\infty} \frac{t^{-\frac{1}{2}} \mathrm{e}^{-t} Y^{(1)}(t)}{1-t / z} \mathrm{~d} t
$$

valid on the Stokes line $\theta=0$. (With the choice of $\theta_{0}$ and $C^{(-1)}\left(\theta_{0}\right)$ made just above (69), the solution $Y^{(1)}(z)$ is still defined by (53).) The close similarity between the integrands of (71) and (60) should be noted. To discuss the asymptotic behavior of $y^{(-1)}(z)$, we follow the calculations presented in $\S 3.3$. The asymptotic expansion of $y^{(-1)}(z)$, valid in $-\pi / 2<\theta<\pi / 2$, is

$$
y^{(-1)}(z) \sim 2 \frac{\mathrm{e}^{z}}{z^{\frac{1}{2}}} \sum_{r=0}^{\infty} \frac{b_{r}}{z^{r}}
$$

where the coefficients $b_{r}$ are still given by (62). The remainder at optimal truncation, after $N$ terms, is approximated by

$$
\frac{\mathrm{e}^{-z}}{z^{\frac{1}{2}}} i \sum_{s=0} \frac{(-1)^{s} b_{s}}{z^{s}}\left[-\operatorname{erf}\left(c^{(1)} \sqrt{\frac{N}{2}}\right)\right]
$$

near $\theta=0$ (as in (42) and (43)) where $c^{(1)}$ is given by $(22)$ with $p^{(-1,1)}=2$. The remainder (73) thus exhibits the Berry smoothing of the Stokes discontinuity across the Stokes line $\theta=0$ of the kind discussed below (43) (in this problem, $c^{(1)} \approx-\theta$ ).

The continuation formula (40) for $y^{(n)}(z)$ is, in this case,

$$
y^{(-1)}\left(z \mathrm{e}^{2 \pi i}\right)=-y^{(-1)}(z)+Y^{(1)}\left(z \mathrm{e}^{2 \pi i}\right)-Y^{(1)}(z) .
$$

From (70), which expresses $y^{(-1)}$ in terms of $\mathrm{Bi}$, and

$$
-\mathrm{e}^{\frac{2}{3} \pi i} \operatorname{Bi}\left(Z \mathrm{e}^{\frac{2}{3} \pi i}\right)=-i \operatorname{Ai}(Z)+i \mathrm{e}^{-\frac{2}{3} \pi i} \operatorname{Ai}\left(Z \mathrm{e}^{-\frac{2}{3} \pi i}\right)
$$


(see Abramowitz and Stegun [1], equation (10.4.6)), from which we can express the last two terms of (74) in terms of $\mathrm{Bi}$, this is equivalent to the known connection formula

$$
\mathrm{e}^{-\frac{2}{3} \pi i} \operatorname{Bi}\left(Z \mathrm{e}^{-\frac{2}{3} \pi i}\right)+\operatorname{Bi}(Z)+\mathrm{e}^{\frac{2}{3} \pi i} \operatorname{Bi}\left(Z \mathrm{e}^{\frac{2}{3} \pi i}\right)=0
$$

(Abramowitz and Stegun [1], equation (10.4.8)).

\section{Bessel functions}

In this section, we illustrate the general results by reference to the modified Bessel differential equation,

$$
z^{2} \frac{\mathrm{d}^{2} Y}{\mathrm{~d} z^{2}}+z \frac{\mathrm{d} Y}{\mathrm{~d} z}-\left(z^{2}+\nu^{2}\right) Y=0
$$

The standard pair of linearly independent solutions of this equation are $K_{\nu}(z)$ and $I_{\nu}(z)$. The functions $K_{ \pm \nu}\left(z \mathrm{e}^{ \pm \pi i}\right)$ and $I_{ \pm \nu}\left(z \mathrm{e}^{ \pm \pi i}\right)$ are also solutions. All of these functions enjoy integral representations which have a common integrand, and which differ only in their contour of integration. These integral representations, originally due to Schläfi (Watson [22], § 6.22), are the basis for our discussion.

We begin by setting out integral representations of particular solutions which we shall need to use. The modified Bessel function $K_{\nu}(z)$ enjoys the integral representation

$$
K_{\nu}(z)=\frac{1}{2} \int_{-\infty}^{\infty} \mathrm{e}^{-z \cosh w} \mathrm{e}^{\nu w} \mathrm{~d} w
$$

when $\theta=0$ (Watson [22], $\S 6.22$, equation (5)). Simple transformations yield representations for the functions $K_{\nu}\left(\mathrm{e}^{ \pm \pi i}\right)$,

$$
K_{\nu}\left(z \mathrm{e}^{\pi i}\right)=\frac{\mathrm{e}^{-\nu \pi i}}{2} \int_{-\infty+i \pi}^{\infty+i \pi} \mathrm{e}^{-z \cosh w} \mathrm{e}^{\nu w} \mathrm{~d} w
$$

when $\theta=-\pi$, and

$$
K_{\nu}\left(z \mathrm{e}^{-\pi i}\right)=\frac{\mathrm{e}^{\nu \pi i}}{2} \int_{-\infty-i \pi}^{\infty-i} \pi \mathrm{e}^{-z \cosh w} \mathrm{e}^{\nu w} \mathrm{~d} w
$$

when $\theta=\pi$.

It is readily shown that any integral of

$$
\mathrm{e}^{-z \cosh w} \mathrm{e}^{\nu w}
$$

(the integrand in (78)-(80)) over a contour which begins and ends at infinity must be a solution of (77). Such contour integrals usually are ascribed in the literature to Sommerfeld (e.g., Henrici [16], p. 344). In the notation of $\S 2$, we define

$$
p(w)=\cosh w, \quad q(w)=\mathrm{e}^{\nu w} .
$$

Our discussions in $\S 2$ and $\S 3$, therefore, apply directly, provided the conditions on $p(w)$ and $q(w)$ referred to in the paragraph between (3) and (4) are satisfied. The conditions hold good provided $|\operatorname{Re}(\nu)|<\frac{1}{2}$ (Boyd [7], p. 511), and we shall assume that $\nu$ satisfies this inequality. We consider other values of $\nu$ at the end of the section.

As in Boyd [7], $\S 6$, where we discussed the asymptotic behavior of the integral representation (78) of $K_{\nu}(z)$, we find that there are an infinite number of saddle 
points at $w^{(n)}=i n \pi$, for $n=0, \pm 1, \pm 2, \ldots$. The steepest-descents path $C^{(n)}(\theta)$ through the saddle point $w^{(n)}$ satisfies the conditions

$$
\begin{aligned}
& \operatorname{Im}\left(z\left(\cosh w-\cosh w^{(n)}\right)\right)=0, \\
& \operatorname{Re}\left(z\left(\cosh w-\cosh w^{(n)}\right)\right) \geq 0 .
\end{aligned}
$$

The paths of steepest descents have the following specific properties.

(i) Each saddle point, say $w^{(n)}$, has two adjacent saddle points, namely $w^{(n-1)}$ and $w^{(n+1)}$; the corresponding adjacent contours are the lines $\operatorname{Im}(w)=(n \pm 1) \pi$, parallel to the real axis (Figure 4). The paths of steepest descents $C^{(n)}(\theta)$ lie between these upper and lower boundaries.

(ii) The set of points comprising the contours $C^{(n)}(\theta)$, when translated by $-2 n \pi i$, is the same as for $C^{(0)}(\theta)$ or $C^{(1)}(\theta)$ according as $n$ is even or odd, respectively.

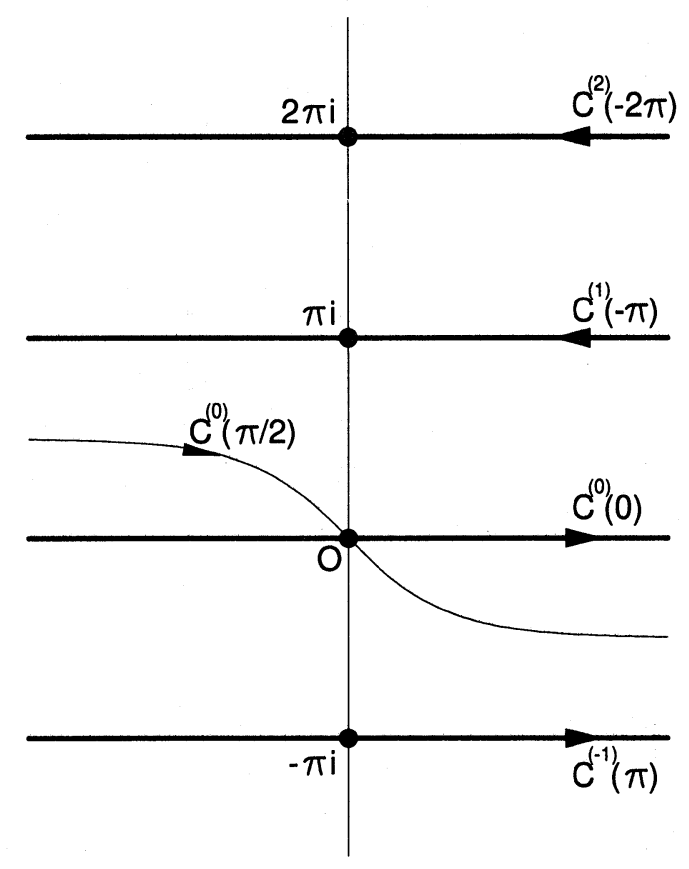

Figure 4. The contours $C^{(-1)}(\pi), C^{(0)}(0), C^{(1)}(-\pi)$ and $C^{(2)}(-2 \pi)$, specified in (86), (84), (85) and (103). Also shown is the contour $C^{(0)}(\pi / 2)$. (The contour $C^{(1)}(\theta)$ is re-defined above $(100)$.)

The general discussion in $\S 3.1$ shows that the infinite number of saddle points divide into two solution classes. In the present case, it follows from (ii) above that the saddle points $w^{(0)}$ and $w^{(1)}$ may be taken to represent their respective solution classes.

To apply the results of $\S 3.2$, let $n=0, \theta_{0}=0$, and specify the orientation of the contour $C^{(0)}\left(\theta_{0}\right)$ by requiring that it be traversed from left to right. Then $m=1,-1$, 
and $-\theta^{(n m)}=-\pi, \pi$, respectively. We consider the solution $Y^{(0)}(z)$, defined to be the integral

$$
Y^{(0)}(z)=\int_{C^{(0)}(\theta)} \mathrm{e}^{-z \cosh w} \mathrm{e}^{\nu w} \mathrm{~d} w
$$

when $\theta=\theta_{0}=0$ (where $C^{(1)}(0)$ is traversed from left to right), and the solutions $Y^{(1)}(z)$ and $Y^{(-1)}(z)$, defined to be the integrals

$$
Y^{(1)}(z)=\int_{C^{(1)}(\theta)} \mathrm{e}^{-z \cosh w} \mathrm{e}^{\nu w} \mathrm{~d} w
$$

when $\theta=-\pi$ (where $C^{(1)}(-\pi)$ is traversed from right to left), and

$$
Y^{(-1)}(z)=\int_{C^{(-1)}(\theta)} \mathrm{e}^{-z \cosh w} \mathrm{e}^{\nu w} \mathrm{~d} w
$$

when $\theta=\pi$ (where $C^{(-1)}(\pi)$ is traversed from left to right). For other values of $\theta$, the solutions $Y^{(0)}, Y^{(1)}(z)$ and $Y^{(-1)}(z)$ are defined by analytic continuation. In particular, $Y^{(0)}(z)$ is represented by the formula (84) for $-\pi<\theta<\pi, Y^{(1)}(z)$ is represented by (85) for $-2 \pi<\theta<0$, and $Y^{(-1)}(z)$ is represented by (86) for $0<\theta<$ $2 \pi$. One can show easily that $\left\{Y^{(0)}(z), Y^{(1)}(z)\right\}$ constitutes a numerically satisfactory pair in $-3 \pi / 2<\theta<\pi / 2$, and that $\left\{Y^{(0)}(z), Y^{(-1)}(z)\right\}$ constitutes a numerically satisfactory pair in $-\pi / 2<\theta<3 \pi / 2$. One readily identifies the solutions $Y^{(0)}(z)$, $Y^{(1)}(z)$ and $Y^{(-1)}(z)$ with standard solutions of the modified Bessel equation:

$$
Y^{(-1)}(z)=2 \mathrm{e}^{-\nu \pi i} K_{\nu}\left(z \mathrm{e}^{-\pi i}\right), \quad Y^{(0)}(z)=2 K_{\nu}(z), \quad Y^{(1)}(z)=-2 \mathrm{e}^{\nu \pi i} K_{\nu}\left(z \mathrm{e}^{\pi i}\right) .
$$

The asymptotic properties of $Y^{(0)}(z)$ and $Y^{(1)}(z)$ may be found from their integral representations (9) of Stieltjes transform type. Considerable simplification is achieved by noting that $Y^{(1)}$ and $Y^{(-1)}$ may be expressed in terms of $Y^{(0)}$. One readily shows that

$$
Y^{(1)}(z)=-\mathrm{e}^{\nu \pi i} Y^{(0)}\left(z \mathrm{e}^{\pi i}\right)
$$

and that

$$
Y^{(-1)}(z)=\mathrm{e}^{-\nu \pi i} Y^{(0)}\left(z \mathrm{e}^{-\pi i}\right) .
$$

Then, the integral representation (9) for $Y^{(0)}(z)$ may be written

$$
Y^{(0)}(z)=\frac{\mathrm{e}^{-z}}{z^{\frac{1}{2}}} \frac{\cos (\nu \pi)}{\pi} \int_{0}^{\infty} \frac{t^{-\frac{1}{2}} \mathrm{e}^{-t} Y^{(0)}(t)}{1+t / z} \mathrm{~d} t,
$$

valid for $-\pi<\theta<\pi$. (The representation (90) agrees with that given in the standard tables of integral transforms of Erdélyi et al. ([15], vol. 2, p. 230, equation (39)).) We now proceed in a similar fashion as we did with (60). Thus, we find the asymptotic expansion

$$
Y^{(0)}(z) \sim \frac{\mathrm{e}^{-z}}{z^{\frac{1}{2}}} \sum_{r=0}^{\infty} \frac{(-1)^{r} b_{r}}{z^{r}}
$$

as $|z| \rightarrow \infty$ in $-3 \pi / 2<\theta<3 \pi / 2$ where the coefficients $b_{r}$ are given by

$$
b_{r}=\frac{\cos (\nu \pi)}{\pi} \int_{0}^{\infty} t^{r-\frac{1}{2}} \mathrm{e}^{-t} Y^{(0)}(t) \mathrm{d} t=\frac{\pi^{\frac{1}{2}}\left(\frac{1}{2}+\nu\right)_{r}\left(\frac{1}{2}-\nu\right)_{r}}{2^{r-\frac{1}{2}} r !} .
$$


The latter result may be found in Boyd ([6], Appendix A) and agrees with that given by Erdélyi et al. ([15], vol. 1, p. 331, equation (28)). We remark that if $\nu$ is real (so that $\left.-\frac{1}{2}<\nu<\frac{1}{2}\right)$, all the coefficients $b_{r}$ are positive. For large $r$, we find that the coefficients are approximated by (cf. (19))

$$
b_{r} \sim \frac{\cos (\nu \pi)}{\pi} \sum_{s=0}^{\infty}(-1)^{s} \frac{\Gamma(r-s)}{2^{r-s}} b_{s} .
$$

The remainder after optimal truncation in (91), say after $N$ terms, may be approximated by

$$
2 i \cos (\nu \pi) \frac{\mathrm{e}^{z}}{z^{\frac{1}{2}}} \sum_{s=0} \frac{b_{s}}{z^{s}}\left[-\frac{1}{2} \operatorname{erfc}\left(-c^{(1)} \sqrt{\frac{N}{2}}\right)\right]
$$

for large $|z|, \theta$ near $-\pi$, and where $c^{(1)}$ is given by $(22)$ with $p^{(01)}=-2$. In this problem, we have

$$
c^{(1)} \approx-(\theta+\pi)
$$

The asymptotic expansions of $Y^{(1)}(z)$ (found from (88) and (91)) and $Y^{(-1)}(z)$ (found from (89) and (91)) are

$$
Y^{(1)}(z) \sim i \mathrm{e}^{\nu \pi i} \frac{\mathrm{e}^{z}}{z^{\frac{1}{2}}} \sum_{s=0}^{\infty} \frac{b_{s}}{z^{s}}, \quad Y^{(-1)}(z) \sim i \mathrm{e}^{-\nu \pi i} \frac{\mathrm{e}^{z}}{z^{\frac{1}{2}}} \sum_{s=0}^{\infty} \frac{b_{s}}{z^{s}}
$$

when $-5 \pi / 2<\theta<\pi / 2$ and $-\pi / 2<\theta<5 \pi / 2$, respectively. We find that (94) exhibits the Berry smoothing of the Stokes discontinuity across the Stokes line $\theta=-\pi$ of the kind discussed below (26) (that is, the exponentially small contributions from (96) are "switched on" as $\theta$ decreases below $-\pi$ ).

The general continuation formula (30), in the special case of the function $Y^{(0)}(z)$, is

$$
Y^{(0)}\left(z \mathrm{e}^{2 \pi i}\right)=-Y^{(0)}(z)+Y^{(-1)}\left(z \mathrm{e}^{2 \pi i}\right)-Y^{(1)}(z),
$$

or, from (88) and (89),

$$
Y^{(0)}\left(z \mathrm{e}^{2 \pi i}\right)=-Y^{(0)}(z)+2 \cos (\nu \pi) Y^{(0)}\left(z \mathrm{e}^{\pi i}\right),
$$

that is to say,

$$
K_{\nu}\left(z \mathrm{e}^{2 \pi i}\right)=-K_{\nu}(z)+2 \cos (\nu \pi) K_{\nu}\left(z \mathrm{e}^{\pi i}\right) .
$$

This is a well-known continuation formula for the modified Bessel function $K_{\nu}(z)$ (Boyd [6], equation (2)).

Now let us consider the solution of Bi type associated with the Stokes line $\theta=0$. To apply the results of $\S 3.3$, we take $n=1, \theta_{0}=-\pi$, and specify the orientation of the contour $C^{(1)}\left(\theta_{0}\right)$ by requiring that it be traversed from left to right (in contrast with our earlier definition below (85)). In the notation of $\S 3.3, m_{+}=0, m_{-}=2$, and $\theta^{\left(n m_{+}\right)}=0$. The definition of $(35)$ for $y^{(1)}(z)$ is

$$
y^{(1)}(z)=\left\{\int_{C^{(1)}(0-)}+\int_{C^{(1)}(0+)}\right\} \mathrm{e}^{-z \cosh w} \mathrm{e}^{\nu w} \mathrm{~d} w
$$

when $\theta=0$; elsewhere, $y^{(1)}(z)$ is defined by analytic continuation. We infer from the representations (79) and (80) that $y^{(1)}(z)$ may be expressed in terms of $K_{\nu}\left(z \mathrm{e}^{\pi i}\right)$ and $K_{\nu}\left(z \mathrm{e}^{-\pi i}\right)$ :

$$
y^{(1)}(z)=2 \mathrm{e}^{\nu \pi i}\left\{K_{\nu}\left(z \mathrm{e}^{\pi i}\right)-K_{\nu}\left(z \mathrm{e}^{-\pi i}\right)\right\}
$$


To derive (101), analytically continue from $\theta= \pm \pi$ to $\theta=0$ in (79) and (80) (a simple change of variable is required for the latter).

The integral representation of Hilbert transform type for $y^{(1)}(z)$ is

$$
y^{(1)}(z)=\frac{\mathrm{e}^{z}}{z^{\frac{1}{2}}} \frac{1}{\pi i}\left\{P \int_{0}^{\infty} \frac{t^{-\frac{1}{2}} \mathrm{e}^{-t} Y^{(0)}(t)}{1-t / z} \mathrm{~d} t-P \int_{0}^{\infty} \frac{t^{-\frac{1}{2}} \mathrm{e}^{-t} Y^{(2)}\left(t \mathrm{e}^{-2 \pi i}\right)}{1-t / z} \mathrm{~d} t\right\},
$$

valid on the Stokes line $\theta=0$. In (102), the solution $Y^{(2)}(z)$ is defined to be

$$
Y^{(2)}(z)=\int_{C^{(2)}(\theta)} \mathrm{e}^{-z \cosh w} \mathrm{e}^{\nu w} \mathrm{~d} w
$$

when $\theta=-2 \pi$ (where $C^{(2)}(-2 \pi)$ is traversed from right to left). With the choice of $\theta_{0}$ and $C^{(1)}\left(\theta_{0}\right)$ made just above (100), the solution $Y^{(0)}(z)$ in (102) is still defined by (84). It may be shown that (cf. (88) and (89))

$$
Y^{(2)}(z)=-\mathrm{e}^{2 \nu \pi i} Y^{(0)}\left(z \mathrm{e}^{2 \pi i}\right)
$$

and so (102) simplifies to

$$
y^{(1)}(z)=\frac{\mathrm{e}^{z}}{z^{\frac{1}{2}}} \frac{2 \mathrm{e}^{\nu \pi i} \cos (\nu \pi)}{\pi i} P \int_{0}^{\infty} \frac{t^{-\frac{1}{2}} \mathrm{e}^{-t} Y^{(0)}(t)}{1-t / z} \mathrm{~d} t .
$$

The asymptotic expansion of $y^{(1)}(z)$, valid for $-\pi / 2<\theta<\pi / 2$, is

$$
y^{(1)}(z) \sim\left(-2 i \mathrm{e}^{\nu \pi i}\right) \frac{\mathrm{e}^{z}}{z^{\frac{1}{2}}} \sum_{r=0}^{\infty} \frac{b_{r}}{z^{r}}
$$

where the coefficients $b_{r}$ continue to be defined by (92). The remainder at optimal truncation, after $N$ terms, is approximated by

$$
\left(2 \mathrm{e}^{\nu \pi i} \cos (\nu \pi)\right) \frac{\mathrm{e}^{-z}}{z^{\frac{1}{2}}} \sum_{s=0} \frac{(-1)^{s} b_{s}}{z^{s}}\left[-\operatorname{erf}\left(c^{(0)} \sqrt{\frac{N}{2}}\right)\right]
$$

near $\theta=0$ where $c^{(0)}$ is given by (22) with $p^{(10)}=2$. In the sector $-7 \pi / 2<\theta<-\pi / 2$, the asymptotic expansion of $Y^{(2)}(z)$ (found from (104) and (91)) is

$$
Y^{(2)}(z) \sim \mathrm{e}^{2 \nu \pi i} \frac{\mathrm{e}^{-z}}{z^{\frac{1}{2}}} \sum_{s=0}^{\infty} \frac{(-1)^{s} b_{s}}{z^{s}}
$$

Together with the asymptotic expansion (91)) for $Y^{(0)}(z)$ in $-3 \pi / 2<\theta<3 \pi / 2$, this result shows that (107) exhibits the Berry smoothing of the Stokes discontinuity across the Stokes line $\theta=0$ of the kind discussed below (43): the exponentially small terms switching signs across the Stokes line (in this case, $c^{(0)} \approx-\theta$ ).

Finally, we illustrate the continuation formula $(40)$ for $y^{(n)}(z)$. It takes the form

$$
y^{(1)}\left(z \mathrm{e}^{2 \pi i}\right)=-y^{(1)}(z)+Y^{(0)}\left(z \mathrm{e}^{2 \pi i}\right)-Y^{(0)}(z)-Y^{(2)}(z)+Y^{(2)}\left(z \mathrm{e}^{-2 \pi i}\right),
$$

which, from (87), (101), and (104), may be expressed

$$
\begin{aligned}
\left\{K_{\nu}\left(z \mathrm{e}^{3 \pi i}\right)-K_{\nu}\left(z \mathrm{e}^{\pi i}\right)\right\}=- & \left\{K_{\nu}\left(z \mathrm{e}^{\pi i}\right)-K_{\nu}\left(z \mathrm{e}^{-\pi i}\right)\right\} \\
& +2 \cos (\nu \pi)\left(K_{\nu}\left(z \mathrm{e}^{2 \pi i}\right)-K_{\nu}(z)\right) .
\end{aligned}
$$

The validity of this result can be verified by using the continuation formula (99).

Our discussion in this section has been restricted to $|\operatorname{Re}(\nu)|<\frac{1}{2}$. If this condition is not satisfied, an alternative approach is feasible under appropriate circumstances. This is discussed in Boyd [7], p. 512. 


\section{Discussion}

We remark first that our results in $\S 2$ (for example on the Berry smoothing, and analytic continuation formulae) are valid generally for integral representations of the form (1), and are not restricted to those which are solutions of differential equations. So too are some of the results in $\S 3.3$, on functions of Bi type associated with a Stokes line.

In the context of differential equations, it may be of interest to generalise our discussion in $\S 3$ to higher-order differential equations, or to second-order differential equations with a large parameter rather than independent variable: in both cases, one readily sees that some results generalise directly, and others do not.

As we remarked earlier, our results in $\S 3.3$ should be capable of extension to the solutions of quite general linear second-order differential equations. In (34), we defined the solution of $\mathrm{Bi}$ type associated with the Stokes line $\theta=-\theta^{(n m)}$ in terms of the integrals $J_{-}$and $J_{+}$. (For simplicity, we suppose that $w^{(n)}$ has only an adjacent saddle on the right, which we denote by $w^{(m)}$.) Now $J_{-}$and $J_{+}$may be regarded as the analytic continuations, to the Stokes line $\theta=-\theta^{(n m)}$, of the solutions which are recessive on the Stokes lines $\theta=-\theta^{(n m)}-\pi$ and $\theta=-\theta^{(n m)}+\pi$, respectively. From (20) and (24), we find that these solutions are formally given by

$$
\frac{\mathrm{e}^{-z p^{(n)}}}{z^{\frac{1}{2}}} \sum_{r=0}^{N-1} \frac{a_{r}}{z^{r}}+\frac{\mathrm{e}^{-z p^{(m)}}}{z^{\frac{1}{2}}} \sum_{s=0}^{\infty} \frac{a_{s}^{(m)}}{z^{s}}\left[-i(-1)^{N-s} F_{N-s}\left(z p^{(n m)} \mathrm{e}^{ \pm \pi i}\right)\right]
$$

near the Stokes line $\theta=-\theta^{(n m)}$ where the $\mathrm{e}^{ \pm \pi i}$ factors in the arguments of $F_{N-s}$ refer to $J_{\mp}$, respectively. Adding the formal representations (111) for $J_{-}$and $J_{+}$, and using (128), then yields

$$
2 \frac{\mathrm{e}^{-z p^{(n)}}}{z^{\frac{1}{2}}} \sum_{r=0}^{N-1} \frac{a_{r}}{z^{r}}+\frac{\mathrm{e}^{-z p^{(m)}}}{z^{\frac{1}{2}}} \sum_{s=0}^{\infty} \frac{a_{s}^{(m)}}{z^{s}}\left[2 i \bar{F}_{N-s}\left(z p^{(n m)}\right)\right],
$$

in agreement with (42). This calculation is purely formal: however, rigorous results of the form (111) have been derived for quite general linear second-order differential equations (Olde Daalhuis and Olver [18]). The formal derivation of (112) from (111), which we have just given is independent of any special integral representations, suggesting that the work of Olde Daalhuis and Olver could be extended to include solutions of $\mathrm{Bi}$ type.

The essential aspect of the discussion we have just given is that, in a rigorous theory, the remainder at optimal truncation should enable one to distinguish between different dominant solutions for the class of asymptotic expansions we are considering (with late coefficients having growth such as (19)). Moreover, one may note that subtracting the formal representations (111) for $J_{-}$and $J_{+}$of the dominant solutions, and using (130), yields the recessive solution. Our work, therefore, suggests that, in the neighbourhood of a Stokes line, the solutions of a linear second-order differential equation can be distinguished by the behavior of their remainders after the contributions from the dominant terms (up to optimal truncation) in their asymptotic approximations have been removed. Specifically, we are suggesting that the solution space of the differential equation in question is isomorphic to that of the canonical differential equation

$$
\frac{\mathrm{d}^{2} v}{\mathrm{~d} x^{2}}+2 x \frac{\mathrm{d} v}{\mathrm{~d} x}=0
$$

whose standard solutions are $1, \operatorname{erfc}( \pm x), \operatorname{erf}(x)$. In the neighbourhood of the Stokes line under consideration, these correspond respectively to the recessive solution, to the 
two solutions which are recessive on the contiguous Stokes lines, and to the solution of Bi type associated with the Stokes line. For example, in the neighbourhood of the Stokes line $\operatorname{ph}(Z)=0$ of the Airy differential equation (45) considered in $\S 4$, these solutions are $\operatorname{Ai}(Z), \operatorname{Ai}\left(Z \mathrm{e}^{ \pm \frac{2}{3} \pi i}\right)$, and $\operatorname{Bi}(Z)$.

Costin and Kruskal [12] have found another means by which the solutions of a linear second-order differential equation can be distinguished in the asymptotic limit. They introduced the notion of the solution of a differential equation being optimally asymptotic to formal asymptotic series (essentially the best asymptotic fit in the solution space). Using this notion, they showed how, for each value of $\theta$, the true and formal solutions could be identified uniquely with each other (their Theorem 1.1). Their conclusions (their $\S 2$ is of particular interest here) are consistent with our remarks above.

\section{Appendix. The terminant functions $F_{p}(z)$ and $\bar{F}_{p}(z)$}

Terminant functions arise when one estimates the remainder which occurs after an asymptotic expansion has been terminated. The idea is due to Dingle: a definition substantially the same as (114) below is given by him in his book ([14], p. 407, equation (22)).

Olver ([21], equation (2.9)) defined the terminant function $F_{p}(z)$, for $\operatorname{Re}(p)>0$, by

$$
F_{p}(z)=\frac{1}{2 \pi} \frac{\mathrm{e}^{-z}}{z^{p-1}} \int_{0}^{\infty} \frac{t^{p-1} \mathrm{e}^{-t}}{t+z} \mathrm{~d} t
$$

when $|\theta|<\pi / 2$, and elsewhere by analytic continuation. (As in the main text, we use the notation $\theta=\operatorname{ph}(z)$.) In earlier work, Olver [20] had defined a terminant function by

$$
T_{p}(z)=\frac{\mathrm{e}^{p \pi i}}{2 \pi i} \frac{\mathrm{e}^{-z}}{z^{p-1}} \int_{0}^{\infty} \frac{t^{p-1} \mathrm{e}^{-t}}{t+z} \mathrm{~d} t
$$

a definition which differs from (114) only by a constant factor. Although the choice of normalisation constant in (115) is convenient for $z$ near the Stokes line $\theta=\pi$, it is inconvenient for $z$ near the Stokes line $\theta=-\pi$. Definition (114) yields results which are symmetric with respect to the two lines (Olver [21], § 2.4, (iii)).

Olver [20] gave asymptotic expansions, elementary and uniform, for $T_{p}(z)$ when $|z|$ was large and $p \approx|z|$. Subsequently, Olver [21] gave expansions for $F_{p}(z)$ in these circumstances: in particular, he gave an expansion which was uniformly valid in a sector which included the Stokes line $\theta=\pi$, and another which was uniformly valid in a sector which included the Stokes line $\theta=-\pi$ (p. 1473, Theorem 1). The latter expansion is obtained by applying complex conjugate operations on the former. The resulting expressions themselves involve complex conjugates, and are not appropriate for our purposes below. Instead, we state the results using notation which is substantially the same as that introduced in an earlier paper (Boyd [6], Appendix B).

Specifically, we take $p$ to be a large positive parameter, and change the variable of integration in (114) from $t$ to $w$ by

$$
t+z-p \ln (-t / z)=p\left(\frac{1}{2} w^{2}+i c w\right)
$$

where the logarithm is specified to be real when $z<0$, and is defined elsewhere by analytic continuation: it is convenient to cut the $z$-plane along the positive real axis. In (116), both left- and right-hand sides are zero when $t=z$ and $w=0$, respectively; it is to be understood that the position of the pole at $t=-z$ is mapped to $w=0$. The, as yet unspecified, constant $c$ in (116) is determined (to within a \pm sign) by the 
requirement that the position of the saddle point at $t=p$ is mapped to $w=-i c$, that is,

$$
\frac{1}{2} c^{2}=1+z / p+\ln (-z / p)
$$

Effectively, the complex parameter $c$ (which depends on the ratio $z / p$ ) measures the proximity of the saddle at $t=p$ and pole at $t=-z$. We choose that branch of $c(z / p)$ whose Taylor expansion about $z / p=-1$ begins $^{1}$

$$
c=-i(z / p+1)-\frac{1}{3} i(z / p+1)^{2}-\frac{7}{36} i(z / p+1)^{3}+\cdots
$$

Now apply the change of variable (116) to the integral (114). Recall that we have cut the $z$-plane along the positive real axis: we consider two cases - when $0<\theta<2 \pi$ and when $-2 \pi<\theta<0$. We find that

$$
F_{p}(z)=-\frac{1}{2 \pi} \frac{\mathrm{e}^{p \ln (-z)}}{z^{p}} \int_{-\infty}^{\infty} \mathrm{e}^{-p\left(\frac{1}{2} w^{2}+i c w\right)}\left(\frac{1}{w}+h(c, w)\right) \mathrm{d} w,
$$

in which the contour of integration passes above the pole at $w=0$ when $0<\theta<2 \pi$, and below when $-2 \pi<\theta<0$. The function $h(c, w)$, which is the same in both cases, is analytic at $w=0$. Further detailed, but routine, calculations then yield asymptotic expansions in the form

$$
F_{p}\left(x \mathrm{e}^{ \pm \pi i}\right) \sim i \mathrm{e}^{\mp p \pi i}\left[ \pm \frac{1}{2} \operatorname{erfc}\left( \pm c \sqrt{\frac{p}{2}}\right)-i \frac{\mathrm{e}^{-\frac{1}{2} p c^{2}}}{\sqrt{2 \pi p}} \sum_{s=0}^{\infty} \frac{h_{s}(c)}{p^{s}}\right]
$$

as $p \rightarrow \infty$ for $|\operatorname{ph}(x)|<\pi$, and where $-x$ replaces $z$ in the previous formulae for $c$. The upper or lower signs in (120) must be used consistently. These results were proved by Olver ([21], Theorem 1, p. 1473); actually he showed that the results are valid for $p-|x|$ bounded in the sector $|\operatorname{ph}(x)| \leq 2 \pi-\delta$ for arbitrarily small positive $\delta$, and that this interval is maximal. As we have stated, our notation differs from Olver's: to recover our results from his, the convention (121) below should be used.

Although the case $p>0$ is that most commonly encountered in applications of $F_{p}(z)$ to the improvement of asymptotic expansions, there is no difficulty in extending (120) to complex $p$ : the result will be valid for $|p|-|x|$ bounded as $|p| \rightarrow \infty$ in the sector $|\operatorname{ph}(x / p)| \leq 2 \pi-\delta$.

The coefficients $h_{s}(c)$ in (120) can be found from the Taylor expansion of $h(c, w)$ about the saddle point $w=-i c$. The coefficients $h_{s}(c)$ in (120) can be derived from those given by Olver ([21], equations (5.10), (5.6). (2.15), (2.16)), provided the following conventions are used (Olver's notation is the left column):

$$
\begin{aligned}
\left(\frac{1}{2}\right)_{s} g_{2 s}(\theta, \alpha) 2^{s} & : \rightarrow h_{s}(c) \\
c(\theta) & : \rightarrow c(x / p) \\
\mathrm{e}^{i \theta} & : \rightarrow-x / p \\
\rho & : \rightarrow p \\
n & : \rightarrow 0 \\
\alpha & : \rightarrow 0
\end{aligned}
$$

\footnotetext{
${ }^{1}$ This choice of $c$ differs from that used by Olver [20] and Boyd [6], but is consistent with that used by Olver [21].
} 
We thus determine

$$
h_{s}(c)=1 \cdot 3 \cdot 5 \ldots(2 s-1)\left[\frac{x}{p} \sum_{j=0}^{2 s}(-1)^{j} \lambda_{2 s-1, j-1}(x / p-1)^{-j-1}+i(-1)^{s+1} c^{-2 s-1}\right] \text {. }
$$

Here, $\lambda_{k, l}$ is the coefficient of $u^{k}$ in the Maclaurin expansion of $[t(u)]^{l}$ where

$$
\frac{1}{2} u^{2}=t-\ln (1+t)
$$

The Maclaurin expansion of $t(u)$ begins

$$
t=u+\frac{1}{3} u^{2}+\frac{1}{36} u^{3}-\frac{1}{270} u^{4}+\frac{1}{4320} u^{5}+\cdots
$$

(Olver [21], equation (2.17)). We thus arrive at the following explicit expressions for the first three coefficients:

$$
\begin{aligned}
h_{0}(c)= & \frac{x}{p}(x / p-1)^{-1}-i c^{-1} \\
h_{1}(c)= & \frac{x}{p}\left[\frac{1}{12}(x / p-1)^{-1}+(x / p-1)^{-3}\right]+i c^{-3}, \\
h_{2}(c)=\frac{x}{p}\left[\frac{1}{288}(x / p-1)^{-1}+\frac{1}{12}(x / p-1)^{-3}\right. & \left.-2(x / p-1)^{-4}+3(x / p-1)^{-5}\right]-3 i c^{-5} .
\end{aligned}
$$

In equations (122) and (125), the right-hand sides are replaced by their limiting values when $c=0$ (and so $x / p=1$ ). For the three coefficients in (125), the Taylor expansions about $x / p=1$ begin

$$
\begin{aligned}
& h_{0}(c)=\frac{2}{3}-\frac{1}{6}(x / p-1)+\cdots \\
& h_{1}(c)=\frac{23}{270}-\frac{143}{288}(x / p-1)+\cdots \\
& h_{2}(c)=\frac{23}{3024}-\frac{97339}{51840}(x / p-1)+\cdots .
\end{aligned}
$$

The tedium of calculating results such as those in (126) can be eased by using a computer algebra system such as (for example) Maple, an introduction to which is given by Char et al. [10].

In the present paper, we need to define a second terminant function, $\bar{F}_{p}(x)$, which is used in special, but commonly-occurring, circumstances. We define $\bar{F}_{p}(x)$, for $\operatorname{Re}(p)>0$, by

$$
\bar{F}_{p}(x)=\frac{1}{2 \pi} \frac{\mathrm{e}^{x}}{x^{p-1}} P \int_{0}^{\infty} \frac{t^{p-1} \mathrm{e}^{-t}}{t-x} \mathrm{~d} t
$$

when $x>0$, and elsewhere by analytic continuation; in (127), $P$ denotes the Cauchy principal value. Equivalently,

$$
\bar{F}_{p}(x)=-\frac{1}{2}\left(\mathrm{e}^{p \pi i} F_{p}\left(x \mathrm{e}^{\pi i}\right)+\mathrm{e}^{-p \pi i} F_{p}\left(x \mathrm{e}^{-\pi i}\right)\right) .
$$

Definition (127) is substantially the same as that given by Dingle ([13], p. 407, equation (23)), though it should be noted that Dingle employs $\bar{\Lambda}(-x)$ where we use $\bar{F}(x)$. Note that the overbar on the $F$ does not denote complex conjugate. 
Application of the asymptotic expansions (120) to the identity (128) yields

$$
\bar{F}_{p}(x) \sim \frac{1}{2} i \operatorname{erf}\left(c \sqrt{\frac{p}{2}}\right)-\frac{\mathrm{e}^{-\frac{1}{2} p c^{2}}}{\sqrt{2 \pi p}} \sum_{s=0}^{\infty} \frac{h_{s}(c)}{p^{s}}
$$

as $p \rightarrow \infty$ for $|\operatorname{ph}(x)| \leq 2 \pi-\delta$ (from Theorem 1 of Olver [21], referenced below (120)). It should be noted that, appearances notwithstanding, both terms in (129) are real when $x$ is positive: for the first term, note that in this circumstance $c$ is purely imaginary; for the second, note that $h(c, w)$ in (119) is real when $z<0$ and $w$ is real.

We remark that result (129) was effectively that employed by Berry [3] in his pioneering work on smoothing across Stokes lines (see Berry's equations (27) and (34), which treated Dawson's integral and the Airy function of the second kind, $\operatorname{Bi}(z)$, respectively.) He considered $c$ to be small, and in this circumstance only the error function term in (129) is significant at leading order. (To be more precise, he effectively used a local approximation for small $c$ : that obtained by taking the first term in the Taylor expansion (3.5) of Olver [21], analogous to our expansion (118).) We discuss the asymptotic behavior of $\operatorname{Bi}(z)$ near the positive real axis, using (129), in $\S 4$.

For reference, we record continuation formulae for $F_{p}(x)$ and $\bar{F}_{p}(x)$. One may derive the formula

$$
\mathrm{e}^{p \pi i} F_{p}\left(x \mathrm{e}^{\pi i}\right)-\mathrm{e}^{-p \pi i} F_{p}\left(x \mathrm{e}^{-\pi i}\right)=i
$$

from (2.11) of Olver [21], or directly from (114). The corresponding formula for $\bar{F}_{p}(x)$,

$$
\mathrm{e}^{p \pi i} \bar{F}_{p}\left(x \mathrm{e}^{\pi i}\right)-\mathrm{e}^{-p \pi i} \bar{F}_{p}\left(x \mathrm{e}^{-\pi i}\right)=\sin (p \pi),
$$

follows from (128) and (130).

\section{Acknowledgement}

Most of this work was done at the Isaac Newton Institute for Mathematical Sciences in Cambridge while the author was a participant in the Exponential Asymptotics programme, held in the first half of 1995. The support of the Institute is acknowledged.

\section{References}

1. M. Abramowitz and I. A. Stegun, Handbook of Mathematical Functions, Dover, New York, 1965.

2. C. M. Bender and S. A. Orszag, Advanced Mathematical Methods for Scientists and Engineers, McGraw-Hill, New York, 1978.

3. M. V. Berry, Uniform asymptotic smoothing of Stokes' discontinuities, Proc. R. Soc. Lond. A 422 (1989), 7-21.

4. M. V. Berry and C. J. Howls, Hyperasymptotics for integrals with saddles, Proc. R. Soc. Lond. A 434 (1991), 657-675.

5. N. Bleistein and R. A. Handelsman, Asymptotic Expansions of Integrals, Holt, Rinehart and Winston, New York, 1975.

6. W. G. C. Boyd, Stieltjes transforms and the Stokes phenomenon, Proc. R. Soc. Lond. A 429 (1990), 227-246.

7. $\frac{1}{403}$ Error bounds for the method of steepest descents Proc. R. Soc. Lond. A 440 (1993), 493-518.

8. __ Gamma function asymptotics by an extension of the method of steepest descents, Proc. R. Soc. Lond. A 447 (1994), 609-630.

9. _ـ Asymptotic approximations for the late coefficients in asymptotic expansions arising in the method of steepest descents, Methods and Applications of Analysis 2 (1995).

10. B. W. Char, K. O. Geddes, G. H. Gonnet, B. L. Leong, M. B. Monagan, and S. M. Watt, First Leaves: A Tutorial Introduction to Maple V, Springer-Verlag, New York, 1992.

11. E. T. Copson, Asymptotic Expansions, Cambridge University Press, Cambridge, 1965.

12. O. Costin and M. D. Kruskal, Optimal uniform estimates and rigorous asymptotics beyond all orders for a class of ordinary differential equations, Proc. R. Soc. Lond. A, 1996 (in press). 
13. N. G. de Bruijn, Asymptotic Methods in Analysis, North Holland, Amsterdam, 1958.

14. R. B. Dingle, Asymptotic Expansions: Their Derivation and Interpretation, Academic Press, London, 1973.

15. A. Erdélyi, W. Magnus, F. Oberhettinger, and F. Tricomi, Tables of Integral Transforms, Vol. 1 and 2, McGraw-Hill, New York, 1954.

16. P. Henrici, Applied and Computational Complex Analysis, Vol. 2. John Wiley and Sons, New York, 1977.

17. H. Jeffreys, Asymptotic Approximations, Oxford University Press, Oxford, 1962.

18. A. B. Olde Daalhuis and F. W. J. Olver, Exponentially improved asymptotic solutions of ordinary differential equations. II Irregular singularities of rank one, Proc. R. Soc. Lond. A 445 (1994), 39-56.

19. F. W. J. Olver, Asymptotics and Special Functions, Academic Press, New York, 1974.

20. _ On Stokes' phenomenon and converging factors, In: Proc. Int. Symp. on Asymptotic and Computational Analysis, Manitoba, Winnipeg, 1989 (Ed. R. Wong), pp.329-355, Marcel Dekker, New York, 1990.

21. __ Uniform, exponentially improved, asymptotic expansions for the generalized exponential integral, SIAM J. Math. Anal. 22 (1991), 1460-1474.

22. G. N, Watson, A Treatise on the Theory of Bessel Eunctions (2nd edition), Cambridge University Press, Cambridge, 1944.

23. D. V. Widder, The Laplace Transform, Princeton University Press, 1941.

24. R. Wong, Asymptotic Approximations of Integrals, Academic Press, New York,1989.

School of Mathematics, University of Bristol, Bristol BS8 1TW

E-mail: w.boyd@bristol.ac.uk 